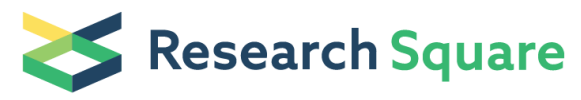 \\ Preprints are preliminary reports that have not undergone peer review. \\ They should not be considered conclusive, used to inform clinical practice, or referenced by the media as validated information.
}

\section{Anticonvulsant Activity, Molecular Modeling and Synthesis of Spirooxindole-4H-Pyran Derivatives using a Novel Reusable Organocatalyst}

\author{
Leila Emami \\ Shiraz University of Medical Sciences School of Pharmacy \\ Leila Moezi \\ Shiraz Medical School: Shiraz University of Medical Sciences \\ Leila Amiri-Zirtol \\ Shiraz Medical School: Shiraz University of Medical Sciences \\ Fatemeh Pirsalami \\ Shiraz Medical School: Shiraz University of Medical Sciences \\ Masoumeh Divar \\ Shiraz University of Medical Sciences School of Pharmacy \\ Aida Solhjoo \\ Shiraz University of Medical Sciences School of Pharmacy \\ Soghra Khabnadideh ( $\sim$ khabns@sums.ac.ir) \\ Shiraz University of Medical Sciences School of Pharmacy
}

\section{Research Article}

Keywords: Anticonvulsant, Spirooxindole-4H-pyran, Graphene Oxide, 1-(2-Aminoethyl) piperazine, Molecular docking,

Pentylenetetrazole, Mice

Posted Date: September 10th, 2021

DOl: https://doi.org/10.21203/rs.3.rs-880526/v1

License: (a) This work is licensed under a Creative Commons Attribution 4.0 International License. Read Full License

Version of Record: A version of this preprint was published at Molecular Diversity on January 25th, 2022. See the published version at https://doi.org/10.1007/s11030-021-10372-7. 


\section{Abstract}

Fifteen derivatives of spirooxindole-4H-pyran $\left(A_{1}-A_{15}\right)$ were subjected to evaluate through intravenous infusion of pentylenetetrazole (PTZ) induced epilepsy mouse models. Four doses of the compounds $(20,40,60,80 \mathrm{mg} / \mathrm{kg})$ were tested in comparison to diazepam as positive control. The resulted revealed that compounds $A_{3}$ and $A_{12}$ were the most active compounds and indicated significant anticonvulsant activity in the PTZ test. The tested compounds were prepared via a multicomponent reaction using graphene oxide (GO) based on the 1-(2-aminoethyl) piperazine as a novel heterogeneous organocatalyst. The prepared catalyst (GO-A.P.) was characterized using some diverse microscopic and spectroscopic procedures as well. The results showed high catalytic activity of the catalyst in the synthesis of spirooxindole- $4 H$-pyran derivatives. The GO-A.P. catalyst was reusable at least for 5 times with no significant decrease in its catalytic action. In silico assessment of physico chemical activity of all compounds also were done which represented appropriate properties. Finally, molecular docking study was performed to achieve their binding affinities as $\gamma$-aminobutyric acid-A (GABA-A) receptor agonists as a plausible mechanism of their anticonvulsant action. Binding free energy values of the compounds represented strongly matched with biological activity.

\section{Introduction}

Epilepsy or seizure is a neurological disorder that involved more than 60 million people in the world (1). Currently, anticonvulsant drugs can control only $70 \%$ of patients and the other patients are resistant to these drugs (2). On the other hand, the present drugs indicated adverse effects such as ataxia, hepatotoxicity, gingival hyperplasia, and megaloblastic anemia and also have a narrow therapeutic window (3). Therefore, the design and synthesis of novel anti-anticonvulsant agents with safety profiles and proper performance are an interesting topic for researchers in medicinal chemistry. Besides, the synthesis of $\mathrm{N}$-containing heterocycles with immense biological activities is a great deal of chemical and biological researchers (4). Spirooxindole systems are of great interest in organic and medicinal chemistry in their broad and promising activities in various therapeutic areas. There has been an intense investigation of different classes of spirooxindole-4H-pyran compounds and many of them were found to be pharmacologically active $(5,6)$. These compounds have recently attracted much attention as a significant category of heterocyclic having useful biological and pharmacological properties such as antibacterial (7), antifungal (8), antitumor (9), antitumor (10), antihypertension (11), and antialzheimer activities (12). In addition, isatin (1 H-indole-2,3-dione) is an endogenous compound that possesses a wide range of biological activities such as anxiogenic, sedative, and anticonvulsant activities. $N$-substituted isatin have also been repeatedly used as intermediates and synthetic precursors for the preparation of a wide variety of heterocyclic compounds with diverse biological activities $(13,14)$. For example some derivatives of isatin have been reported to have anticonvulsant activities (15). Many synthetic methodologies were reported for synthesizing of spiro-cycles including 2-oxindole based on cycloaddition or multicomponent reactions (MCRs) $(16,17)$. Some catalysts such as sodium stearate (18), L-proline (19), triethylbenzylammonium chloride (TEBA) (20), InCl3 (21), and SBA-Pr- $\mathrm{NH}_{2}$ (22) have been used for the synthesis of spirooxindole$4 H$-pyran. But there is still demand for inexpensive catalyst and nontoxic organic solvents with the green chemistry protocols. Today, the use of the organocatalyst is substantial interest in current-day research. Due to the numerous advantages over conventional transition-metal catalysis, organocatalytic methodologies have become an attractive synthetic tool in organic synthesis (23). In this regard, carbon nanomaterial especially graphene oxide (GO) through by large theoretical specific surface area, high intrinsic mobility, thermal conductivity and non-toxicity have attracted considerable attention as an organocatalyst (24). The presence of several groups like epoxy, hydroxyl, carboxyl and carbonyl on GO are well recognized and accepted as major functionalities attached to it (25). Generally, reduced graphene oxide is used if water dispersibility is desired, however it has the drawbacks of having higher sheet resistance and lower carrier mobility than even highly grained monolayer graphene sheets (26). The GO mainly consists of individual sheets of graphene with attachments of oxygen functional groups on both the basal planes and edges (25). Nitrogen-containing compounds have excellently complexing properties due to their abundant amino functional groups (27). In order to obtain an innovative adsorbent, functionalized GO with 1-(2-aminoethyl)-piperazine has been developed. 1(2-aminoethyl)-piperazine was used to modify GO to opening its epoxide rings. By this means, the distance between the GO layers was increased and also provides greater and easier access to the catalyst. The catalyst was documented using different spectroscopic techniques. We also described the catalytic activity of our catalyst in the one pot synthesis of spirooxindole- $4 H$-pyran derivatives. Using of GO as a solid support with high surface area, high yield catalytic reactions in a mild condition and being a reusable catalyst is the other advantages of our catalyst. In order to investigate the anticonvulsant activities of the spirooxindole$4 H$-pyran derivatives, all the prepared compounds were evaluated in the PTZ test. Besides, a plausible mechanism for

Page 2/19 
anticonvulsant activity was described as $\gamma$-aminobutyric acid (GABA) agonist. The GABA-A is an ion channel receptor and GABA identified as an inhibitory neurotransmitter which is widely distributed throughout the neuraxis (28-31). GABA is considered to be a multifunctional molecule that has different situational functions and involved in reducing neuronal excitability in the central nervous system (32). In this regard, molecular docking simulation was applied to find out their binding conformations and structural specificities of these compounds as a GABA-A receptor agonist. The physicochemical and ADMET properties were also calculated for all compounds.

\section{Results And Discussion}

GO was prepared according to the Hummers method. 1-(2-Aminoethyl) piperazine in ethanol was then exposed to GO at reflux temperature in ethanol without any activator. Epoxide rings and carboxylic acids are potent functional groups on the surface of $\mathrm{GO}$ for reaction to 1-(2-aminoethyl) piperazine. Assignation of an organic compound on the surface of $\mathrm{GO}$ causes to create a gap between its layers and increasing their distances which leads to improve the catalytic activity. Nucleophilic properties of 1-(2aminoethyl) piperazine makes it suitable as a catalyst in MCR reactions. After preparation the catalyst it was considered with different analytic methods. The catalytic activity of GO-A.P. was then established by its application in the synthesis of spirooxindole-4H-pyran derivatives $\left(A_{1}-A_{15}\right)$. The reaction conditions were also optimized in the point of temperature, solvent and amounts of catalyst. After spending the catalyst in the chemical reactions it could be separate and reused for 5 times without any significant decrease in its catalytic capacity.

\section{Catalyst Identification}

\section{FT-IR analysis}

Comparing of FT-IR spectrums of GO before and after functionalization with 1-(2-aminoethyl) piperazine specifies the presence of different functional groups on its surface. The primary FT-IR spectrum of GO showed a broad peak at $3357 \mathrm{~cm}^{-1}$ which is related to the vibration's intensity of $\mathrm{OH}$ groups. Decreasing of this peak in GO-A.P. spectra is proving good replacing of these groups with other moieties. The sharp peak in $2814 \mathrm{~cm}^{-1}$ which is belongs to the $\mathrm{CH}_{2}$ groups of piperazine ring is another evidence to the presence of 1-(2-aminoethyl) piperazine in the catalyst structure. However, the main differences between the IR spectrum before and after functionalization are seen in the range of 1000 to $1700 \mathrm{~cm}^{-1}$ which are related to the $\mathrm{C}-\mathrm{N}, \mathrm{CH}_{2}$, epoxide, alkoxide, carboxyl/carbonyl $(\mathrm{C}=0)$ and aromatic $\mathrm{C}=\mathrm{C}$ bonds in the catalyst (Fig. 1).

\section{Raman spectroscopy}

Structure regularity of the catalyst was confirmed by Raman spectroscopy. Vibration modes of $s p 3$ and $s p 2$ carbon atoms are presented as D and G peaks respectively. The ID / IG ratio is an expression of irregularities in carbon structures and introduction of $s p 3$ defects. This ratio is about 0.92 for $\mathrm{GO}$ which is increased to 1.02 for functionalized GO-A.P. Development in the ID / IG ratio indicates the entry of $s p 3$ carbon atoms into the $\mathrm{GO}$ plates via binding to 1-(2-aminoethyl) piperazine (Fig. 2).

\section{XRD studies}

Distances between GO layers could be determined by XRD spectra. XRD patterns of GO demonstrate a sharp peak at $2 \theta=10.375^{0}$, which represents the distance of $0.8 \mathrm{~A}^{\circ}$ between its layers. Introducing of 1-(2-aminoethyl) piperazine to the GO, creates a gap between the sheets and causes this peak to be appear at $2 \theta=6.7^{0}$, which shows the distance of $1.3 \mathrm{~A}^{\circ}$ between the layers (Fig. 3). The heightening in the distance between the GO-A.P. layers advises the successful connection of organic functional groups to the GO surface.

\section{EDS analysis}

The percentages of $\mathrm{C}, \mathrm{N}$, and $\mathrm{O}$ in the GO-A.P. composite were detected as $50.41 \%, 30.27 \%$, and $19.31 \%$, respectively by EDS analysis. These measures are matched to the predicted values in the chemical structure of the composite. Furthermore, the 
presence of nitrogen atoms in EDS analysis is a prominent evidence for the presence of 1-(2-aminoethyl) piperazine in the catalyst structure (Fig. 4).

\section{Optimization of the reaction conditions}

Subsequently, the catalytic activity of GO-A.P. for the synthesis of spirooxindole- $4 H$-pyran derivatives was optimized through the reaction of $\mathrm{N}$-butyl isatin $(1 \mathrm{mmol})$, malononitrile $(0.162 \mathrm{~g}, 1 \mathrm{mmol})$ and 3-hydroxycoumarin $(0.32 \mathrm{~g}, 1 \mathrm{mmol})$ to get $\mathrm{A}_{2}$ as a model reaction. To achieve the most favorable conditions, some parameters such as temperature, solvent and amounts of the catalyst were studied (Table 1). The effectiveness of piperazine on the reaction progress was confirmed through entries $1-3$. In order to investigate the effect of solvent, various media such as ethanol, water and DCM were tested in this regard. The best result was observed by ethanol with $93 \%$ efficiency (Table 1, entries $3-5$ ). In the next step to find the best temperature conditions, the reaction was examined at $70^{\circ} \mathrm{C}$, room temperature and reflux. The reaction efficiency reached $93 \%$ at $70^{\circ} \mathrm{C}$ in 30 minutes (Table 1, entries 8 ). Then the required amount of catalyst was also optimized. The reaction was conducted in different amounts of GO-A.P. (0.01, 0.02, 0.03 , and $0.04 \mathrm{~g}$ ) (Table 1, entries 7-10). The best result was obtained with $0.03 \mathrm{~g}$. Therefore, ethanol at $70^{\circ} \mathrm{C}$ in the presence of $0.03 \mathrm{~g}$ of catalyst was selected as the best condition for synthesis of other analogues.

Table 1

Optimization of the reaction conditions for synthesis of $A_{2}$.

\begin{tabular}{|llllll|}
\hline Entry & Catalyst $(\mathrm{gr})$ & Solvent & Temperature $\left({ }^{\circ} \mathrm{C}\right)$ & Time $(\mathrm{min})$ & Yield (\%) \\
\hline 1 & - & $\mathrm{EtOH}$ & 70 & 35 & 10 \\
\hline 2 & $\mathrm{GO}(0.04)$ & $\mathrm{EtOH}$ & 70 & 40 & 45 \\
\hline 3 & GO.A.P. (0.04) & $\mathrm{EtOH}$ & 70 & 30 & 93 \\
\hline 4 & GO.A.P. (0.04) & $\mathrm{H} \mathrm{H}_{\mathrm{O}}$ & 70 & 90 & 50 \\
\hline 5 & GO.A.P. (0.04) & $\mathrm{DCM}$ & 70 & 90 & - \\
\hline 6 & GO.A.P. (0.04) & $\mathrm{EtOH}$ & Reflux & 30 & 93 \\
\hline 8 & GO.A.P. (0.04) & $\mathrm{EtOH}$ & r.t & $4 \mathrm{~h}$ & 60 \\
\hline 9 & GO.A.P. (0.03) & $\mathrm{EtOH}$ & 70 & 30 & 93 \\
\hline 10 & GO.A.P. (0.02) & EtOH & 70 & 80 & 75 \\
\hline
\end{tabular}

Catalytic activity of the catalyst

After optimization of the reaction conditions, the effectiveness of GO-A.P. was proved by one-pot synthesis reaction among various spirooxindole-4H-pyran $\left(A_{1}-A_{15}\right)$ derivatives. The results showed this catalytic system was quite efficient for various isatin analogues. In these spiro compounds all isatin derivatives were successfully converted to the desired products with high-efficiency yields (Table 2). Nucleophilic properties of the amine groups in piperazine ring have critical role in a nucleophilic attack to the epoxide groups in the surface of $\mathrm{GO}$ result in creating a heterogeneous catalyst. This heterogeneous catalyst expresses both acidic and basic properties. The plausible mechanistic pathways for the synthesis of spiropyrane compounds with available catalyst are shown in Fig. 5. 
Table 2

Chemical structures, melting points and yields of the final synthesized Spirooxindole- $4 H$-pyran derivatives

\begin{tabular}{|c|c|c|c|c|}
\hline Entry & Chemical name & Spectra data & $\begin{array}{l}\text { m.p. } \\
\left({ }^{\circ} \mathrm{C}\right) \\
\text { Obs/ } \\
\text { Lit. } \\
(16)\end{array}$ & $\begin{array}{l}\text { Yield } \\
(\%)\end{array}$ \\
\hline$A_{1}$ & $\begin{array}{l}\text { 2'-Amino-1-ethyl- } \\
\text { 2,5' -dioxo-5' H-- } \\
\text { spiro[indoline-3,4' } \\
\text {-pyrano[3,2- } \\
\text { c]chromene]-3' - } \\
\text { carbonitrile }\end{array}$ & 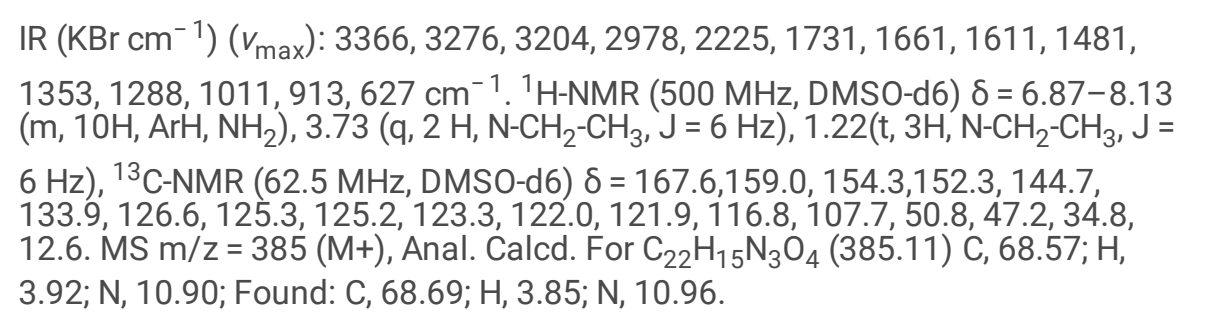 & $\begin{array}{l}253- \\
255 / \\
255- \\
257\end{array}$ & 94 \\
\hline $\mathrm{A}_{2}$ & $\begin{array}{l}\text { 2'-Amino-1-butyl- } \\
\text { 2,5'-dioxo-5'H- } \\
\text { spiro[indoline-3,4'- } \\
\text { pyrano[3,2- } \\
\text { c]chromene]-3'- } \\
\text { carbonitrile }\end{array}$ & $\begin{array}{l}\text { IR }\left(\mathrm{KBr} \mathrm{cm}^{-1}\right)\left(v_{\mathrm{max}}\right): 3361,3196,2992,2206,1643,1464,1075,973,753 \mathrm{~cm}^{-1} \text {. } \\
{ }^{1} \mathrm{H}-\mathrm{NMR}(300 \mathrm{MHz}, \mathrm{DMSO}-\mathrm{d} 6) \delta=6.90-7.91\left(\mathrm{~m}, 10 \mathrm{H}, \mathrm{ArH} \mathrm{NH}_{2}\right), 4.05-4.12(\mathrm{~m}, \\
\left.2 \mathrm{H}, \mathrm{N}-\mathrm{CH}_{2}-\mathrm{CH}_{2}-\mathrm{CH}_{2}-\mathrm{CH}_{3}\right), 1.44-1.57\left(\mathrm{~m}, 4 \mathrm{H}, \mathrm{N}-\mathrm{CH}_{2}-\mathrm{CH}_{2}-\mathrm{CH}_{2}-\mathrm{CH}_{3}\right), 0.94(\mathrm{t}, 3 \mathrm{H}, \mathrm{N}- \\
\left.\mathrm{CH}_{2}-\mathrm{CH}_{2}-\mathrm{CH}_{2}-\mathrm{CH}_{3}, \mathrm{~J}=6 \mathrm{~Hz}\right),{ }^{13} \mathrm{C}-\mathrm{NMR}(62.5 \mathrm{MHz}, \mathrm{DMSO}-\mathrm{d} 6) \delta=175.9,158.9, \\
158.6,155.6,152.5,143.6,134.1,132.8,129.5,125.4,124.4,123.1,117.1,112.9, \\
109.0,101.7,57.3,47.6,29.5,19.9,14.1 . \mathrm{MS} \mathrm{m} / \mathrm{z}=413(\mathrm{M}+), \text { Anal. Calcd. For } \\
\mathrm{C}_{24} \mathrm{H}_{19} \mathrm{~N}_{3} \mathrm{O}_{4}(413.14) \mathrm{C}, 69.72 ; \mathrm{H}, 4.63 ; \mathrm{N}, 10.16 ; \text { Found: } \mathrm{C}, 69.90 ; \mathrm{H}, 4.52 ; \mathrm{N}, \\
10.22 .\end{array}$ & $\begin{array}{l}259- \\
261 / \\
259- \\
260\end{array}$ & 93 \\
\hline $\mathrm{A}_{3}$ & $\begin{array}{l}\text { 2'-Amino-1-(3- } \\
\text { methylbut-2-en-1- } \\
\text { yl)-2,5'-dioxo-5'0H- } \\
\text { spiro[indoline-3,4'- } \\
\text { pyrano[3,2- } \\
\text { c]chromene]- } \\
\text { 3'-carbonitrile }\end{array}$ & $\begin{array}{l}\text { IR }\left(\mathrm{KBr} \mathrm{cm}^{-1}\right)\left(v_{\text {max }}\right): 3350,3255,3186,2932,1604,1363,1198,1111,1027, \\
932,755,540 \mathrm{~cm}^{-1} .{ }^{1} \mathrm{H}-\mathrm{NMR}(300 \mathrm{MHz}, \mathrm{DMSO}-\mathrm{d} 6) \delta=7.97-6.91(\mathrm{~m}, 10 \mathrm{H}, \mathrm{ArH}, \\
\left.\mathrm{NH}_{2}\right), 5.18-5.14(\mathrm{~m}, 1 \mathrm{H}, \mathrm{CH}), 4.42-4.25\left(\mathrm{~m}, 2 \mathrm{H}, \mathrm{CH}_{2}\right), 1.80\left(\mathrm{~s}, 3 \mathrm{H}, \mathrm{CH}_{3}\right), 1.69(\mathrm{~s}, \\
\left.3 \mathrm{H}, \mathrm{CH}_{3}\right),{ }^{13} \mathrm{C}-\mathrm{NMR}(62.5 \mathrm{MHz}, \mathrm{DMSO}-\mathrm{d} 6) \delta=177.1,167.5,159.2,158.2,154.3 \\
154.2,152.3,145.1,135.2,134.4,134.0,133.9,128.5,125.5,125.3,123.3,123.3, \\
122.0,119.4,116.8,112.9,107.9,104.1,75.7,59.2,38.4,25.9,18.3,14.0 . \mathrm{MS} \\
\mathrm{m} / \mathrm{z}=425(\mathrm{M}+), \text { Anal. Calcd. For } \mathrm{C}_{25} \mathrm{H}_{19} \mathrm{~N}_{3} \mathrm{O}_{4}(425.14) \mathrm{C}, 70.58 ; \mathrm{H}, 4.50 ; \mathrm{N}, 9.88 \\
\text { Found: } \mathrm{C}, 71.07 ; \mathrm{H}, 4.47 ; \mathrm{N}, 9.95 .\end{array}$ & $\begin{array}{l}249- \\
251 / \\
249- \\
251\end{array}$ & 91 \\
\hline $\mathrm{A}_{4}$ & $\begin{array}{l}\text { 2'-Amino-1-(3- } \\
\text { bromobenzyl)-2,5'- } \\
\text { dioxo-5'H- } \\
\text { spiro[indoline-3,4'- } \\
\text { pyrano[3,2- } \\
\text { c]chromene]-3'- } \\
\text { carbonitrile }\end{array}$ & 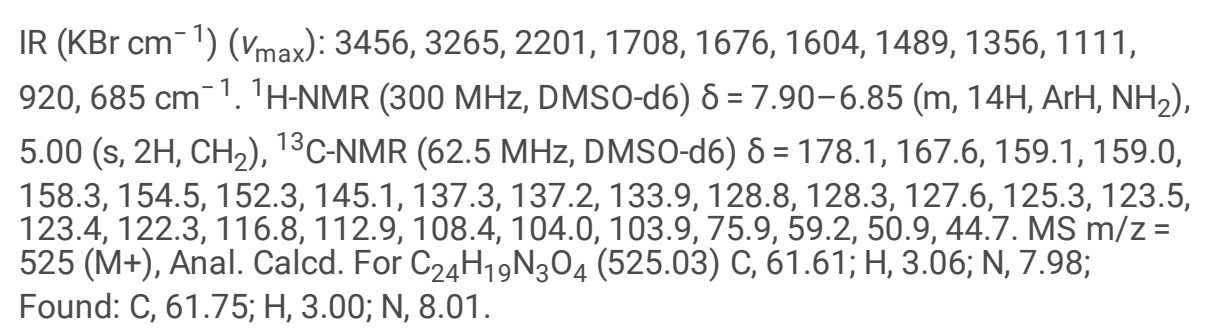 & $\begin{array}{l}267- \\
269 / \\
267- \\
269\end{array}$ & 87 \\
\hline $\mathrm{A}_{5}$ & $\begin{array}{l}\text { 2'-Amino-1-(3- } \\
\text { bromobenzyl)-2,5'- } \\
\text { dioxo-5'H- } \\
\text { spiro[indoline-3,4'- } \\
\text { pyrano[3,2- } \\
\text { c]chromene]-3'- } \\
\text { carbonitrile }\end{array}$ & $\begin{array}{l}\text { IR }\left(\mathrm{KBr} \mathrm{cm}{ }^{-1}\right)\left(v_{\text {max }}\right): 3456,3265,2201,1708,1676,1604,1489,1356,1111, \\
920,685 \mathrm{~cm}^{-1} .{ }^{1} \mathrm{H}-\mathrm{NMR}(300 \mathrm{MHz}, \mathrm{DMSO}-\mathrm{d} 6) \delta=7.90-6.85\left(\mathrm{~m}, 14 \mathrm{H}, \mathrm{ArH}, \mathrm{NH}_{2}\right), \\
5.00\left(\mathrm{~s}, 2 \mathrm{H}, \mathrm{CH}_{2}\right),{ }^{13} \mathrm{C}-\mathrm{NMR}(62.5 \mathrm{MHz}, \mathrm{DMSO}-\mathrm{d} 6) \delta=178.1,167.6,159.1,159.0, \\
158.3,154.5,152.3,145.1,137.3,137.2,133.9,128.8,128.3,127.6,125.3,123.5, \\
123.4,122.3,116.1112 .9,108.4,104.0,103.9,75.9,59.2,50.9,44.7 . \mathrm{MS} \mathrm{m} / \mathrm{z}= \\
525(\mathrm{M}+), \text { Anal. Calcd. For } \mathrm{C}_{24} \mathrm{H}_{19} \mathrm{~N}_{3} \mathrm{O}_{4}(525.03) \mathrm{C}, 61.61 ; \mathrm{H}, 3.06 ; \mathrm{N}, 7.98 ; \\
\text { Found: } \mathrm{C}, 61.75 ; \mathrm{H}, 3.00 ; \mathrm{N}, 8.01 .\end{array}$ & $\begin{array}{l}267- \\
269 / \\
267- \\
269\end{array}$ & 87 \\
\hline$A_{6}$ & $\begin{array}{l}\text { Ethyl-2'-amino-1- } \\
\text { butyl-2,5'-dioxo-5'- } \\
\text { H-spiro[indoline- } \\
\text { 3,4'-pyrano[3,2- } \\
\text { c]chromene]-3'- } \\
\text { carboxylate }\end{array}$ & 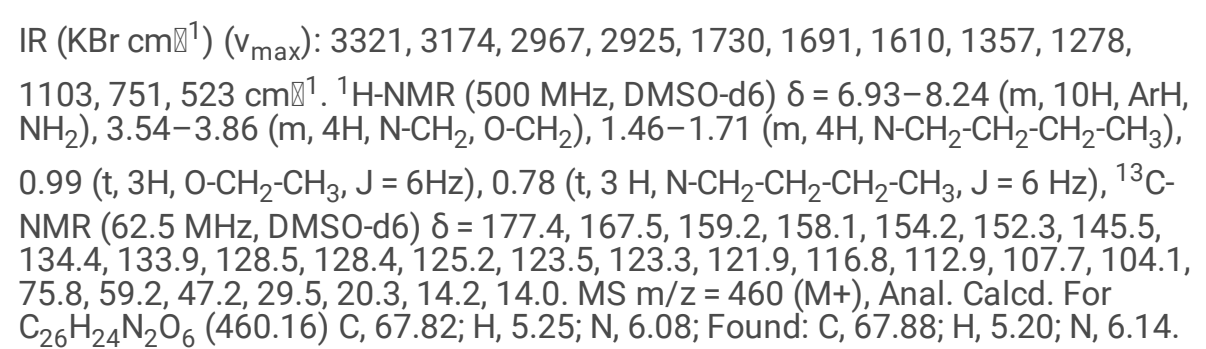 & $\begin{array}{l}218- \\
220 / \\
217- \\
219\end{array}$ & 91 \\
\hline
\end{tabular}




\begin{tabular}{|c|c|c|c|c|}
\hline Entry & Chemical name & Spectra data & $\begin{array}{l}\text { m.p. } \\
\left({ }^{\circ} \mathrm{C}\right) \\
\text { Obs/ } \\
\text { Lit. } \\
(16)\end{array}$ & $\begin{array}{l}\text { Yield } \\
(\%)\end{array}$ \\
\hline$A_{7}$ & $\begin{array}{l}\text { Methyl 2' -amino- } \\
\text { 1-(3-methylbut-2- } \\
\text { en-1-yl)-2,5' - } \\
\text { dioxo-5' H- } \\
\text { spiro[indoline-3,4' } \\
\text {-pyrano[3,2-c] } \\
\text { chromene]-3' - } \\
\text { carboxylate }\end{array}$ & $\begin{array}{l}\text { IR }\left(\mathrm{KBr} \mathrm{cm}^{-1}\right)\left(v_{\text {max }}\right): 3369,3307,3189,2940,1719,1661,1612,1481,1353, \\
1288,1111,1027,989,762 \mathrm{~cm}^{-1} .{ }^{1} \mathrm{H}-\mathrm{NMR}(500 \mathrm{MHz}, \mathrm{DMSO}-\mathrm{d} 6) \delta=6.83-8.19 \\
\left(\mathrm{~m}, 10 \mathrm{H}, \mathrm{ArH}, \mathrm{NH}_{2}\right), 5.28-5.34(\mathrm{~m}, 1 \mathrm{H}, \mathrm{CH}), 4.25-4.33\left(\mathrm{~m}, 2 \mathrm{H}, \mathrm{N}-\mathrm{CH}_{2}\right), 3.35(\mathrm{~s}, \\
\left.3 \mathrm{H}, \mathrm{O}-\mathrm{CH}_{3}\right), 1.81\left(\mathrm{~s}, 3 \mathrm{H}, \mathrm{CH}_{3}\right), 1.71\left(\mathrm{~s}, 3 \mathrm{H}, \mathrm{CH}_{3}\right),{ }^{13} \mathrm{C}-\mathrm{NMR}(62.5 \mathrm{MHz}, \mathrm{DMSO}-\mathrm{d} 6) \delta \\
=177.0,167.7,159.2,159.1,158.2,135.3,134.1,133.9,128.6,128.5,125.5, \\
\text { 125.2 123.5, } 123.3,122.0,119.4,116.8,112.8,107.9,75.8,59.2,50.8,47.3,38.3, \\
\text { 25.9, 18.3. MS m/Z = 458 }(\mathrm{M}+), \text { Anal. Calcd. For } \mathrm{C}_{26} \mathrm{H}_{22} \mathrm{~N}_{2} \mathrm{O}_{6}(458.14) \mathrm{C}, 68.11 ; \\
\mathrm{H}, 4.84 ; \mathrm{N}, 6.11 ; \text { Found: } \mathrm{C}, 68.17 ; \mathrm{H}, 4.80 ; \mathrm{N}, 6.19 .\end{array}$ & $\begin{array}{l}227- \\
229 / \\
227- \\
229\end{array}$ & 86 \\
\hline$A_{8}$ & $\begin{array}{l}\text { Methyl 2' -amino- } \\
\text { 1-cyclopentyl-2,5' - } \\
\text { dioxo-5' H- } \\
\text { spiro[indoline-3,4' } \\
\text {-pyrano[3,2-c] } \\
\text { chromene]- 3' - } \\
\text { carboxylate }\end{array}$ & 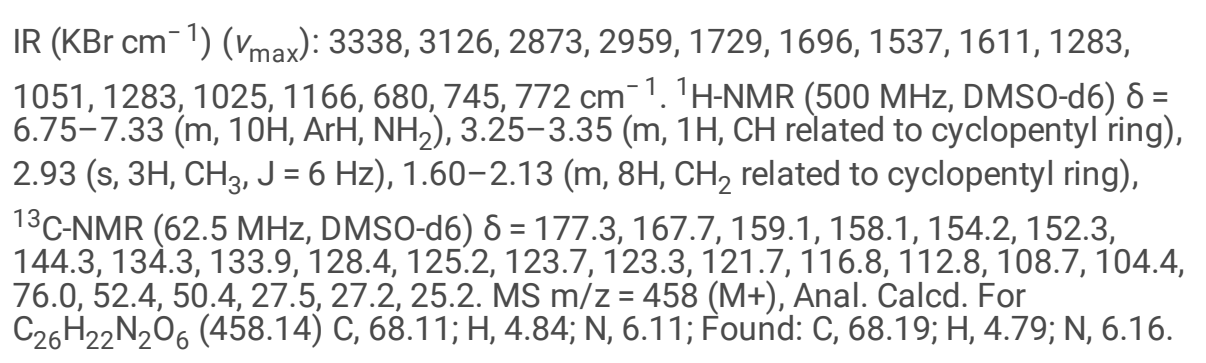 & $\begin{array}{l}255- \\
257 / \\
255- \\
257\end{array}$ & 88 \\
\hline$A_{9}$ & $\begin{array}{l}\text { Methyl 2' -amino- } \\
\text { 1-benzy'-2,5' - } \\
\text { dioxo-5' H- } \\
\text { spiro[indoline-3,4' } \\
\text {-pyrano[3,2-c] } \\
\text { chromene]-3' - } \\
\text { carboxylate }\end{array}$ & $\begin{array}{l}\text { IR }\left(\mathrm{KBr} \mathrm{cm}^{-1}\right)\left(v_{\mathrm{max}}\right): 3379,3270,3200,2940,1972,1719,1661,1612,1481, \\
1353,1288,1111,1027,989,696 \mathrm{~cm}^{-1} .{ }^{1} \mathrm{H}-\mathrm{NMR}(500 \mathrm{MHz}, \mathrm{DMSO}-\mathrm{d} 6) \delta= \\
6.50-7.96\left(\mathrm{~m}, 15 \mathrm{H}, \mathrm{ArH}, \mathrm{NH}_{2}\right), 4.63\left(\mathrm{~s}, 2 \mathrm{H}, \mathrm{CH}_{2}\right), 3.09\left(\mathrm{~s}, 3 \mathrm{H}, \mathrm{CH}_{3}\right),{ }^{13} \mathrm{C}-\mathrm{NMR} \\
(62.5 \mathrm{MHz}, \mathrm{DMSO}-\mathrm{d} 6) \delta=178.1,167.6,159.2,159.0,158.3,154.5,152.3,145.1 \\
137.3,133.9,128.8,128.7,128.5,128.3,127.6,125.3,123.5,123.4,122.3,116.8, \\
112.9,108.4,75.9,59.2,50.9,47.4,44.7 . \mathrm{MS} \mathrm{m} / \mathrm{z}=480(\mathrm{M}+), \text { Anal. Calcd. For } \\
\mathrm{C}_{28} \mathrm{H}_{20} \mathrm{~N}_{2} \mathrm{O}_{6}(480.13) \mathrm{C}, 69.99 ; \mathrm{H}, 4.20 ; \mathrm{N}, 5.83 ; \text { Found: } \mathrm{C}, 70.03 ; \mathrm{H}, 4.16 ; \mathrm{N}, 5.87 .\end{array}$ & $\begin{array}{l}299 \\
- \\
281 / \\
299- \\
281\end{array}$ & 90 \\
\hline $\mathrm{A}_{10}$ & $\begin{array}{l}\text { Ethyl 2' -amino-1- } \\
\text { ethyl-2,5' -dioxo-5' } \\
\text { H-spiro[indoline- } \\
\text { 3,4' -pyrano[3,2- } \\
\text { c]chromene] -3' } \\
\text { carboxylate }\end{array}$ & $\begin{array}{l}\text { IR }\left(\mathrm{KBr} \mathrm{cm}{ }^{-1}\right)\left(v_{\text {max }}\right): 3360,3312,3189,2944,1716,1665,1612,1481,1353, \\
1284,1117,1127,909,662 \mathrm{~cm}^{-1} \cdot{ }^{1} \mathrm{H}-\mathrm{NMR}(500 \mathrm{MHz}, \mathrm{DMSO}-\mathrm{d} 6) \delta=6.86-8.20 \\
\left(\mathrm{~m}, 10 \mathrm{H}, \mathrm{ArH}, \mathrm{NH}_{2}\right), 3.77-3.85\left(\mathrm{~m}, 2 \mathrm{H}, \mathrm{O}-\mathrm{CH}_{2}-\mathrm{CH}_{3}\right), 3.61-3.71\left(\mathrm{~m}, 2 \mathrm{H}, \mathrm{N}-\mathrm{CH}_{2}{ }^{-}\right. \\
\left.\mathrm{CH}_{3}\right), 1.23\left(\mathrm{t}, 3 \mathrm{H}, \mathrm{O}-\mathrm{CH}_{2}-\mathrm{CH}_{3}, \mathrm{~J}=6 \mathrm{~Hz}\right), 0.73\left(\mathrm{t}, 3 \mathrm{H}, \mathrm{N}-\mathrm{CH}_{2}-\mathrm{CH}_{3}, \mathrm{~J}=6 \mathrm{~Hz}\right),{ }^{13} \mathrm{C}- \\
\mathrm{NMR}(62.5 \mathrm{MHz}, \mathrm{DMSO}-\mathrm{d} 6) \delta=177.1,167.5,159.2,158.2,154.2,152.3,144.9, \\
134.4,133.9,128.5,125.2,123.5,123.3,121.9,116.8,112,8,107.6,104.2,75.7 \\
59.2,47.7,17.0,12.4 . \mathrm{MS} \mathrm{m} / \mathrm{z}=432(\mathrm{M}+), \text { Anal. Calcd. For } \mathrm{C}_{24} \mathrm{H}_{20} \mathrm{~N}_{2} \mathrm{O}_{6}(432.13) \\
\mathrm{C}, 66.66 ; \mathrm{H}, 4.66 ; \mathrm{N}, 6.48 ; \text { Found: } \mathrm{C}, 66.71 ; \mathrm{H}, 4.73 ; \mathrm{N}, 6.52\end{array}$ & $\begin{array}{l}243- \\
245 / \\
246- \\
24\end{array}$ & 87 \\
\hline$A_{11}$ & $\begin{array}{l}\text { Methyl 2' -amino- } \\
\text { 1-butyl-2,5' -dioxo- } \\
\text { 5' H-spiro[indoline- } \\
\text { 3,4' -pyrano[3,2-c] } \\
\text { chromene]-3' - } \\
\text { carboxylate }\end{array}$ & 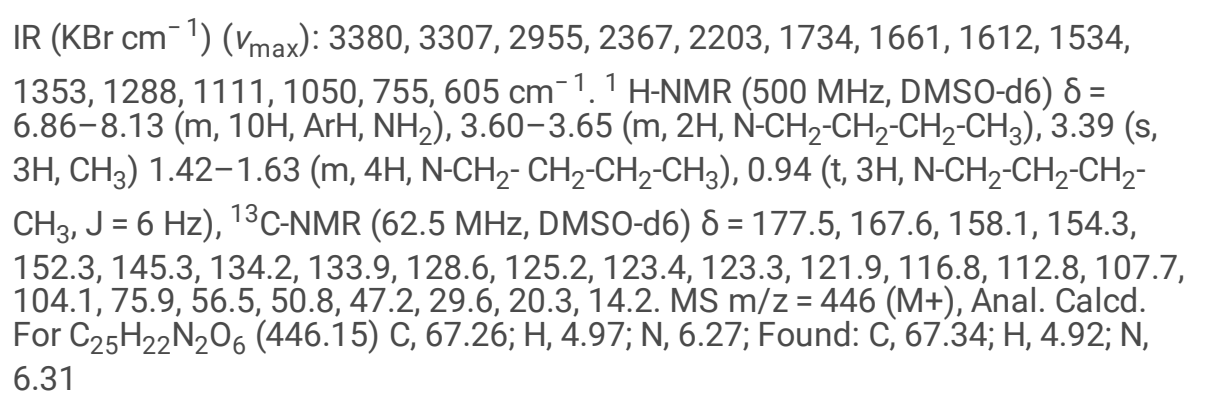 & $\begin{array}{l}208- \\
210 / \\
207- \\
212\end{array}$ & 93 \\
\hline
\end{tabular}




\begin{tabular}{|c|c|c|c|c|}
\hline Entry & Chemical name & Spectra data & $\begin{array}{l}\text { m.p. } \\
\left({ }^{\circ} \mathrm{C}\right) \\
\text { Obs/ } \\
\text { Lit. } \\
(16)\end{array}$ & $\begin{array}{l}\text { Yield } \\
(\%)\end{array}$ \\
\hline$A_{12}$ & $\begin{array}{l}\text { Ethyl 2' -amino-1- } \\
\text { (sec-butyl)-2,5' - } \\
\text { dioxo-5' H- } \\
\text { spiro[indoline-3,4' } \\
\text {-pyrano[3,2-c] } \\
\text { chromene]-3' - } \\
\text { carboxylate }\end{array}$ & 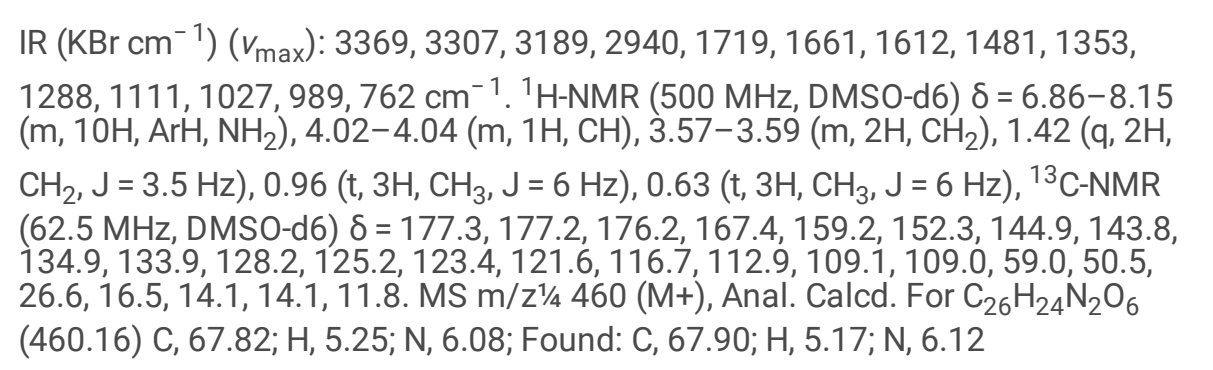 & $\begin{array}{l}234- \\
236 / \\
233- \\
235\end{array}$ & 87 \\
\hline$A_{13}$ & $\begin{array}{l}\text { Ethyl 2' -amino-1- } \\
\text { (3-methylbut-2-en- } \\
\text { 1-yl)-2,5' -dioxo-5' } \\
\text { H-spiro[indoline- } \\
\text { 3,4' -pyrano [3,2- } \\
\text { c]chromene]-3' - } \\
\text { carboxylate }\end{array}$ & $\begin{array}{l}\text { IR }\left(\mathrm{KBr} \mathrm{cm}{ }^{-1}\right)\left(v_{\max }\right): 3332,3121,2870,2953,1729,1696,1537,1611,1283, \\
1051,1283,1025,1166,681,745,772 \mathrm{~cm}^{-1} .{ }^{1} \mathrm{H}-\mathrm{NMR}(500 \mathrm{MHz}, \mathrm{DMSO}-\mathrm{d} 6) \delta= \\
6.83-8.20\left(\mathrm{~m}, 10 \mathrm{H}, \mathrm{ArH}, \mathrm{NH}_{2}\right), 5.28-5.34(\mathrm{~m}, 1 \mathrm{H}, \mathrm{CH}), 3.66-3.84\left(\mathrm{~m}, 2 \mathrm{H}, \mathrm{CH}_{2}\right), \\
1.81\left(\mathrm{~s}, 3 \mathrm{H}, \mathrm{CH}_{3}\right), 1.72\left(\mathrm{~s}, 3 \mathrm{H}, \mathrm{CH}_{3}\right), 0.72\left(\mathrm{t}, 3 \mathrm{H}, \mathrm{CH}_{3}, \mathrm{~J}=6 \mathrm{~Hz}\right),{ }^{13} \mathrm{C}-\mathrm{NMR}(62.5 \\
\mathrm{MHz}, \mathrm{DMSO}-\mathrm{d} 6) \delta=177.1,167.5,159.2,158.2,154.3,154.2,152.3,145.1,135.2 \\
134.4,134.0,133.9,128.5,125.2,123.5,123.3,122.0,119.4,116.8,112.9,107.9, \\
104.1,75.7,59.2,18.3,14.0 . \mathrm{MS} \mathrm{m} / \mathrm{z}=472(\mathrm{M}+), \text { Anal. Calcd. For } \mathrm{C}_{27} \mathrm{H}_{24} \mathrm{~N}_{2} \mathrm{O}_{6} \\
(472.16) \mathrm{C}, 68.63 ; \mathrm{H}, 5.12 ; \mathrm{N}, 5.93 ; \text { Found: } \mathrm{C}, 68.68 ; \mathrm{H}, 5.10 ; \mathrm{N}, 5.97 .\end{array}$ & $\begin{array}{l}225- \\
227 / \\
225- \\
227\end{array}$ & 88 \\
\hline $\mathrm{A}_{14}$ & $\begin{array}{l}\text { Ethyl 2'-amino-1- } \\
\text { cyclopentyl-2,5'- } \\
\text { dioxo-5'H- } \\
\text { spiro[indoline-3,4'- } \\
\text { pyrano[3,2-c] } \\
\text { chromene]-3'- } \\
\text { carboxylate }\end{array}$ & $\begin{array}{l}\text { IR }\left(\mathrm{KBr} \mathrm{cm}^{-1}\right)\left(v_{\text {max }}\right): 3332,3121,2953,2870,1712,1696,1611,1463,1350, \\
1283,1025,970,706,681,572 \mathrm{~cm}^{-1} .{ }^{1} \mathrm{H}-\mathrm{NMR}(300 \mathrm{MHz}, \mathrm{DMSO}-\mathrm{d} 6) \delta=6.75- \\
7.33\left(\mathrm{~m}, 10 \mathrm{H}, \mathrm{ArH}, \mathrm{NH}_{2}\right), 3.25-3.35(\mathrm{~m}, 1 \mathrm{H}, \mathrm{cyclopentyl}), 3.61\left(\mathrm{q}, 2 \mathrm{H}, \mathrm{CH}_{2}, \mathrm{~J}=\right. \\
6 \mathrm{~Hz}), 1.60-2.13(\mathrm{~m}, 8 \mathrm{H}, \mathrm{cyclopentyl}),\left(\mathrm{t}, 3 \mathrm{H}, \mathrm{CH}_{3}, \mathrm{~J}=6 \mathrm{~Hz}\right),{ }^{13} \mathrm{C}-\mathrm{NMR}(62.5 \mathrm{MHz}, \\
\text { DMSO-d6) } \delta=176.5,158.1,158.8,155.7,152.5,143.2,136.3,134.2,132.7 \\
129.4,128.8,127.6,125.5,124.5,123.3,123.2,117.4,117.1,112.9,109.6,101.6, \\
57.3,47.8,43.9 \mathrm{MS} \mathrm{m} / \mathrm{z}=472(\mathrm{M}+), \text { Anal. Calcd. For } \mathrm{C}_{27} \mathrm{H}_{24} \mathrm{~N}_{2} \mathrm{O}_{6}(472.16) \mathrm{C}, \\
68.63 ; \mathrm{H}, 5.12 ; \mathrm{N}, 5.93 ; \text { Found: } \mathrm{C}, 68.69 ; \mathrm{H}, 5.09 ; \mathrm{N}, 5.98 .\end{array}$ & $\begin{array}{l}> \\
300 / \\
278- \\
280\end{array}$ & 87 \\
\hline$A_{15}$ & $\begin{array}{l}\text { Ethyl 2' -amino-1- } \\
\text { benzyl-2,5' -dioxo- } \\
\text { 5' H-spiro[indoline- } \\
\text { 3,4' -pyrano[3,2- c] } \\
\text { chromene]-3' - } \\
\text { carboxylate }\end{array}$ & $\begin{array}{l}\text { IR }\left(\mathrm{KBr} \mathrm{cm}^{-1}\right)\left(v_{\mathrm{max}}\right): 3376,3317,3289,2940,1729,1661,1622,1485,1343, \\
1285,1116,1027,989,765 \mathrm{~cm}^{-1} .{ }^{1} \mathrm{H} \text { NMR }(500 \mathrm{MHz}, \mathrm{DMSO}-\mathrm{d} 6) \delta=6.56-7.96 \\
\left(\mathrm{~m}, 15 \mathrm{H}, \mathrm{ArH}, \mathrm{NH}_{2}\right), 3.66-4.32\left(\mathrm{~m}, 4 \mathrm{H}, \mathrm{CH}_{2}\right), 0.72\left(\mathrm{t}, 3 \mathrm{H}, \mathrm{CH}_{3}, \mathrm{~J}=6 \mathrm{~Hz}\right),{ }^{13} \mathrm{CNMR} \\
(62.5 \mathrm{MHz}, \mathrm{DMSO}-\mathrm{d} 6) \delta=178.1,167.6,159.2,159.0,158.3,154.5,152.3,145.3 \\
145.1,137.3,133.9,128.8,128.3,127.6,125.3,123.6,123.5,123.4,122.3,116.8, \\
112.9,108.4,75.9,59.2,50.9,47.4,44.7 \mathrm{MS} \mathrm{m} / \mathrm{z}=494(\mathrm{M}), \text { Anal. Calcd. For } \\
\mathrm{C}_{29} \mathrm{H}_{22} \mathrm{~N}_{2} \mathrm{O}_{6}(494.15) \mathrm{C}, 70.44 ; \mathrm{H}, 4.48 ; \mathrm{N}, 5.67 ; \text { Found: } \mathrm{C}, 70.49 ; \mathrm{H}, 4.42 ; \mathrm{N}, 5.71\end{array}$ & $\begin{array}{l}230- \\
232 / \\
230- \\
232\end{array}$ & 89 \\
\hline
\end{tabular}

To show the advantage of GO-A.P. over some of the previously reported catalysts in the literature, the catalytic role of GO-A.P. in spirooxindole-4H-pyran synthesis compared with the various reported catalysts. The results show the most capability of our methods (Table 3).

Table 3

Comparison of the yield obtained in this work with other researches for synthesis of spiropyrane derivatives

\begin{tabular}{|llll|}
\hline Entry & Catalyst/Temp/Time/Solvent & Yield (\%) & Ref. \\
\hline 1 & {$\left[\right.$ Dabco-H]Cl $/ 50^{\circ} \mathrm{C} / 2 \mathrm{~h} / \mathrm{CH}_{3} \mathrm{CN}$} & 95 & \\
2 & $\mathrm{BN} @ \mathrm{Fe}_{3} \mathrm{O}_{4} / 80^{\circ} \mathrm{C} / 45 \mathrm{~min} /$ Water & 88 & \\
\hline 3 & $\mathrm{Et}_{3} \mathrm{~N} / 75^{\circ} \mathrm{C} / 45 \mathrm{~min} / \mathrm{EtOH}$ & 90 & \\
\hline 5 & GO-A.P. $/ 70^{\circ} \mathrm{C} / 30 \mathrm{~min} / \mathrm{EtOH}$ & 93 & This work \\
\hline
\end{tabular}




\section{Catalyst recovery and reuse}

The synthesis of $A_{2}$ as a model reaction was done to investigate the recyclability of the GO-A.P. After completion the reaction, the catalyst was separated from the reaction mixture using filtration and washed with hot ethanol. Finally, it dried at room temperature and then used for the next run. As expected, the recycled catalyst could be reused without a considerable decrease in its catalytic activity for 5 runs (Fig. 6).

\section{Anticonvulsant activity of the spirooxindole- $4 \mathrm{H}$-pyran derivatives}

All the synthesized spirooxindole-4H-pyran derivatives were screened for the anticonvulsant properties to distinguish compounds that increase the seizure threshold in PTZ induced seizure model. Different doses of compounds $(20,40,60$ and $80 \mathrm{mg} / \mathrm{kg})$ were administrated and their effects on intravenous PTZ-induced clonic seizure threshold are shown in Table 4. Diazepam (1 mg/kg) was used as positive control. Compounds $A_{3}$ and $A_{12}$ increased the seizure threshold in all doses $(20,40,60 \mathrm{and} 80 \mathrm{mg} / \mathrm{kg})$ significantly. Meanwhile, compounds $A_{2}-A_{4}, A_{9}, A_{12}$ and $A_{14}$ in 40,60 and $80 \mathrm{mg} / \mathrm{kg}$ elevated the seizure threshold in comparison to the solvent group. Also, compounds $A_{2}-A_{4}, A_{6}, A_{9}$ and $A_{11}-A_{14}$ induced anticonvulsant effect in intravenous PTZ model in doses of 60 and $80 \mathrm{mg} / \mathrm{kg}$. Even compound $A_{5}$ induced significant anticonvulsant effect in doses of $20,40 \mathrm{and} 60 \mathrm{mg} / \mathrm{kg}$ but it is inactive in $80 \mathrm{mg} / \mathrm{kg}$. Compounds $A_{7}, A_{10}$, and $A_{15}$ only in dose of $80 \mathrm{mg} / \mathrm{kg}$ enhanced the seizure threshold in comparison to the solvent group. Compounds $A_{1}$ and $A_{8}$ did not change the seizure threshold when compared to the solvent group in all doses (Fig. 7).

Table 4

Effect of spirooxindole-4H-pyran derivatives on the seizure threshold in PTZ-induced seizure model

\begin{tabular}{|c|c|c|c|c|}
\hline & 20 mg/kg & 40 mg/kg & $60 \mathrm{mg} / \mathrm{kg}$ & $80 \mathrm{mg} / \mathrm{kg}$ \\
\hline$A_{1}$ & $34.26 \pm 1.29$ & $36.10 \pm 2.23$ & $38.02 \pm 1.35$ & $37.28 \pm 1.04$ \\
\hline$A_{2}$ & $36.37 \pm 1.02$ & $39.93 \pm 1.39 *$ & $39.46 \pm 1.86 *$ & $43.39 \pm 1.73^{\star \star \star}$ \\
\hline$A_{3}$ & $39.09 \pm 1.46^{*}$ & $44.59 \pm 0.84^{\star \star \star}$ & $39.51 \pm 1.05^{\star \star}$ & $42.91 \pm 1.28 * \star \star$ \\
\hline $\mathrm{A}_{4}$ & $34.38 \pm 2.00$ & $39.19 \pm 0.94 *$ & $39.23 \pm 1.4^{\star \star}$ & $43.95 \pm 1.14^{\star \star \star}$ \\
\hline $\mathrm{A}_{5}$ & $45.54 \pm 1.75^{\star \star \star}$ & $45.85 \pm 1.93^{\star \star \star}$ & $39.45 \pm 1.01 * \star$ & $35.64 \pm 0.89$ \\
\hline$A_{6}$ & $37.05 \pm 1.28$ & $38.70 \pm 1.27$ & $40.88 \pm 2.02 * \star$ & $41.78 \pm 1.61^{\star \star}$ \\
\hline$A_{7}$ & $29.75 \pm 2.25$ & $30.41 \pm 1.91$ & $37.66 \pm 1.26$ & $45.63 \pm 0.95^{\star \star \star}$ \\
\hline$A_{8}$ & $32.24 \pm 36.1$ & $32.08 \pm 2.06$ & $36.40 \pm 0.66$ & $37.80 \pm 1.26$ \\
\hline$A_{9}$ & $36.98 \pm 0.35$ & $40.15 \pm 3.26 *$ & $42.62 \pm 1.93^{\star \star *}$ & $40.98 \pm 0.86 * * \star$ \\
\hline$A_{10}$ & $31.59 \pm 1.02$ & $32.49 \pm 1.24$ & $33.74 \pm 0.46$ & $44.57 \pm 1.21^{\star \star \star}$ \\
\hline$A_{11}$ & $33.13 \pm 0.68$ & $31.17 \pm 1.61$ & $45.86 \pm 1.71 * * \star$ & $43.12 \pm 1.69 * \star \star$ \\
\hline$A_{12}$ & $41.67 \pm 0.8^{\star \star \star}$ & $42.83 \pm 0.66^{\star \star \star}$ & $42.77 \pm 1.49 * \star \star$ & $42.84 \pm 1.23^{\star \star \star}$ \\
\hline$A_{13}$ & $27.4 \pm 1.43$ & $27.6 \pm 1.61$ & $44.27 \pm 1.48^{\star \star \star}$ & $44.27 \pm 1.5^{\star \star \star}$ \\
\hline$A_{14}$ & $38.6 \pm 1.51$ & $46.71 \pm 1.07 * * \star$ & $46.14 \pm 1.37 * \star \star$ & $43.47 \pm 1.28 * \star \star$ \\
\hline$A_{15}$ & $32.52 \pm 0.79$ & $31.42 \pm 2.35$ & $31.77 \pm 1.14$ & 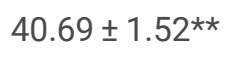 \\
\hline Diazepam & $58.52 \pm 1.88^{* \star *}$ & & & \\
\hline Solvent & $32.81 \pm 1.16$ & & & \\
\hline
\end{tabular}


Drug likeness and in silico physicochemical parameters prediction of the spirooxindole-4H-pyran compounds

In the next step, all the synthesized compounds were subjected to molecular properties prediction and drug-likeness by Swiss ADME and PreADMET software's. Table 5 represented that all compounds have molecular weight in the accepted range $(\mathbb{\square} 500$ $\mathrm{gr} / \mathrm{mol}$ ) except compound $\mathrm{A}_{5}$. In addition, other properties including number of rotatable bonds ( $\mathrm{n}-\mathrm{RB}$ ), HBD and HBA as hydrogen bond properties were within the acceptable limit. This means, the synthesized compounds exhibit desire conformational flexibility. Meanwhile, all compounds have TPSA in the region of 109-112 $A^{0}$, point to good penetration of the blood-brain barrier (BBB). All of the compounds have also acceptable lipophilicity ( $\log P)$ and they obey Lipinski's rule of five.

Table 5

Physicochemical properties of the spirooxindole-4H-pyran compounds

\begin{tabular}{|c|c|c|c|c|c|c|c|}
\hline Entry & $M^{a}$ & $\log \mathrm{P}^{b}$ & $\mathrm{HBD}^{\mathrm{C}}$ & HBA $^{d}$ & $\operatorname{TPSA}\left(\mathrm{A}^{2}\right)^{\mathrm{e}}$ & $n-R^{f}$ & Lipinski violation \\
\hline$A_{1}$ & 358.37 & 1.68 & 1 & 5 & 109.56 & 1 & 0 \\
\hline$A_{2}$ & 413.43 & 2.1 & 1 & 5 & 109.56 & 3 & 0 \\
\hline$A_{3}$ & 425.44 & 2.24 & 1 & 5 & 109.56 & 2 & 0 \\
\hline$A_{4}$ & 425.44 & 2.31 & 1 & 5 & 109.56 & 1 & 0 \\
\hline$A_{5}$ & 526.34 & 3.08 & 1 & 5 & 109.56 & 2 & 0 \\
\hline$A_{6}$ & 446.45 & 2.34 & 1 & 6 & 112.07 & 5 & 0 \\
\hline$A_{7}$ & 458.46 & 2.47 & 1 & 6 & 112.07 & 4 & 0 \\
\hline$A_{8}$ & 458.46 & 2.54 & 1 & 6 & 112.07 & 3 & 0 \\
\hline$A_{9}$ & 480.47 & 2.73 & 1 & 6 & 112.07 & 4 & 0 \\
\hline$A_{10}$ & 432.43 & 2.13 & 1 & 6 & 112.07 & 4 & 0 \\
\hline$A_{11}$ & 460.48 & 2.54 & 1 & 6 & 112.07 & 6 & 0 \\
\hline$A_{12}$ & 460.48 & 2.54 & 1 & 6 & 112.07 & 5 & 0 \\
\hline$A_{13}$ & 472.49 & 2.67 & 1 & 6 & 112.07 & 5 & 0 \\
\hline$A_{14}$ & 472.49 & 2.74 & 1 & 6 & 112.07 & 4 & 0 \\
\hline$A_{15}$ & 494.49 & 2.93 & 1 & 6 & 112.07 & 5 & 0 \\
\hline Diazpam & 284.74 & 2.67 & 0 & 2 & 32.67 & 1 & 0 \\
\hline Rule of Lipinski & $\leq 500$ & $\leq 5$ & $\leq 5$ & $\leq 10$ & $\leq 140$ & $\leq 10$ & $\leq 1$ \\
\hline
\end{tabular}

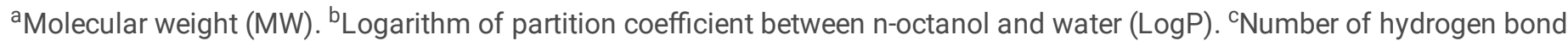
donors (HBD). ${ }^{d}$ Number of hydrogen bond acceptors (HBA). ${ }^{e}$ Topological polar surface area(TPSA). ${ }^{f}$ Number of rotatable bonds $(\mathrm{nRB})$.

The drug-likeness and Absorbance Distribution Metabolism and Excretion (ADME) properties of all compounds were shown in Table 6. Plasma protein binding (PPB) and percentages of human intestinal absorption (HIA) as pharmacokinetic parameters represented that all compounds exhibited high PPB values (more than 80 ) except compound $A_{8}$ which had low value (63).

Regarding HIA, all compounds showed high values, which illustrated high absorption from the intestine to the bloodstream. In vitro Caucasian colon adeno carcinoma cell permeability (Caco-2) value represented that compounds have appropriate permeability for penetration to biological membranes. Also, all of the compounds have BBB value more than 0.1 which represented these derivatives, could consider as CNS agents for epilepsy therapy. 
Table 6

Insilico ADME of the spirooxindole-4H-pyran compounds

\begin{tabular}{|c|c|c|c|c|}
\hline Entry & $\% H I A^{a}$ & Invitro Caco-2 cell permeability ( $\mathrm{nm} \mathrm{s}^{-1}$ ) & $\%$ Invitro plasma protein bonding & $\% \mathrm{BBB}^{\mathrm{b}}$ \\
\hline$A_{1}$ & 97.65 & 20.74 & 79.73 & 0.15 \\
\hline$A_{2}$ & 98.28 & 20.04 & 90.52 & 0.42 \\
\hline$A_{3}$ & 98.32 & 20.71 & 86.22 & 0.44 \\
\hline$A_{4}$ & 98.24 & 18.74 & 86.40 & 0.18 \\
\hline$A_{5}$ & 97.15 & 21.05 & 100 & 0.55 \\
\hline$A_{6}$ & 98.18 & 20.73 & 84.92 & 0.15 \\
\hline$A_{7}$ & 98.33 & 20.71 & 82.26 & 0.26 \\
\hline$A_{8}$ & 97.75 & 19.88 & 63.18 & 0.15 \\
\hline$A_{9}$ & 98.19 & 20.81 & 88.72 & 0.21 \\
\hline$A_{10}$ & 97.98 & 20.8 & 80.74 & 0.18 \\
\hline$A_{11}$ & 98.33 & 20.62 & 86.1 & 0.184 \\
\hline$A_{12}$ & 98.33 & 20.41 & 86.04 & 0.11 \\
\hline$A_{13}$ & 98.45 & 21.18 & 86.25 & 0.33 \\
\hline$A_{14}$ & 98.42 & 19.36 & 86.74 & 0.137 \\
\hline$A_{15}$ & 98.22 & 20.84 & 88.73 & 0.29 \\
\hline Diazpam & 99.49 & 47.68 & 98.74 & 2.58 \\
\hline
\end{tabular}

\section{Molecular docking study}

As it was mentioned maybe the anticonvulsant activity of spirooxindole-4H-pyrans was apply via an agonistic effect on the GABA-A receptors. Hence, molecular docking simulation studies were directed to determine the specific binding site and binding mode of the synthesized compounds to GABA-A receptor as their proposed targets. Molecular docking studies of the synthesized compounds with the GABA-A receptor protein represented desire docking scores (Table 7). The resulted showed that compounds $A_{3}$ and $A_{12}$ which were the most active derivatives in the in vivo study, represented best results in molecular docking studies as well.

Table 7

The bonding energies ( $\mathrm{kcal} / \mathrm{mol}$ ) of the tested compounds on GABA (6X3X) and (4COF) receptors using AutoDock Vina

\begin{tabular}{|c|c|c|c|c|c|c|c|c|c|c|c|c|c|c|c|c|}
\hline Entry & $A_{1}$ & $A_{2}$ & $A_{3}$ & $A_{4}$ & $A_{5}$ & $A_{6}$ & $A_{7}$ & $A_{8}$ & $A_{9}$ & $A_{10}$ & $A_{11}$ & $A_{12}$ & $A_{13}$ & $\mathrm{~A}_{14}$ & $A_{15}$ & $\begin{array}{l}\mathrm{CO} \text { (crystal } \\
\text { ligand) }\end{array}$ \\
\hline $6 \times 3 x$ & -6 & -7.5 & -8.8 & -8 & -8.1 & -6.7 & -7.1 & -7.4 & -7.4 & -6.8 & -6.7 & -8.9 & -7.2 & -7.4 & -6.9 & $\begin{array}{l}-6.7 \\
\text { (Diazpam) }\end{array}$ \\
\hline $4 \mathrm{COF}$ & -7 & -8.3 & -9.8 & -8 & -10 & -7.7 & -8.4 & -8.4 & -8.7 & -8.1 & -7.7 & -9.7 & -8.1 & -8.6 & -8.4 & $\begin{array}{l}-8.6 \\
\text { (Benzamidine) }\end{array}$ \\
\hline
\end{tabular}

As seen in Fig. 8, compound $A_{3}$, presenting both top score and top biological activity, shows a hydrogen bond interaction with Met283. Additionally, this compound involved in pi-pi interaction with Phe331 and also, there is existence pi-alkyl interaction with 
Val290. Compound $A_{1}$ presenting the less biological activity, shows some only pi-alkyl interaction with Val290 and Met283. It is noteworthy that high biological activity compounds formed one or more hydrogen bonds with the active site residues.

As depicted in Fig. 9, the key amino acids in the binding mode of compound $A_{3}$ are Ser 46 and Lue 99, which interact with the compounds by hydrogen bonding between carbonyl group of isatin ring and pi-sigma interaction of phenyl moiety of coumarin respectively. Also some hydrophobic interaction with Ala 145, Ala 135 and Met 137 are also observed. On the other hand, regarding compound, the oxygen atom of pyrane ring is involved in hydrogen bond with Met 137, and there is also existed some hydrophobic interactions with residue Ser46, Arg 180 and Ala 137.

\section{Materials And Methods}

All chemicals were obtained from Merck or Fluka and were used without any additional purification. All yields refer to isolated products after purification methods. The products were characterized by spectroscopic methods and comparison of their physical properties with those reported in the literature. Infrared spectra were run on a Shimadzu FTIR-8300 spectrophotometer. The ${ }^{1} \mathrm{H}-\mathrm{NMR}$ $(300 \mathrm{MHz})$ and ${ }^{13} \mathrm{C}$-NMR $(62.5 \mathrm{MHz})$ were run on a Bruker Advanced DPX-250. Melting points were recorded on a B€uchi B 545 apparatus in open capillary tubes and are uncorrected. Natural graphite powder (diameter 5-10 nm, thickness 4-20 nm, layers $<30$ and purity $>99.5$ wt. \%), was acquired from Neutrino Co. (Iran). Pentylenetetrazole (PTZ) was dissolved in physiological saline solution and DMSO was used as co-solvent. Diazepam was purchased from Kimidaroo (Iran) and was dissolved in normal saline. In all experiments PTZ was administered intravenously (iv) and all other drugs were administered intraperitoneally (ip) 30 min before PTZ.

\section{General procedure for Preparation of Graphene Oxide-1-(2-aminoethyl) piperazine (GO-A.P.)}

Hammer's improved method was used to synthesize GO. First $0.1 \mathrm{~g}$ of $\mathrm{GO}$ was dispersed in EtOH $(10 \mathrm{~mL})$ for 30 minutes and then 1-(2-aminoethyl) piperazine $(0.2 \mathrm{~mL})$ was added. The reaction mixture stirred at reflux temperature for 24 hours. Then the catalyst was separated by centrifugation, washed with ethanol and dried at $60^{\circ} \mathrm{C}$ for 24 hours (Fig. 10).

\section{General procedure for Synthesis of spirooxindole-4 H -pyran derivatives}

$\mathrm{N}$-substituted isatin intermediates prepared as reported previously in the literature. A mixture of $\mathrm{N}$-alkyl or aryl isatin ( $2 \mathrm{mmol})$, nitrile moiety $(0.162 \mathrm{~g}, 2.5 \mathrm{mmol}), 3$-hydroxycoumarin $(0.32 \mathrm{~g}, 2 \mathrm{mmol})$, and GO-A.P. $(0.3 \mathrm{~g})$ in EtOH $(25 \mathrm{~mL})$ were reacted at $70^{\circ} \mathrm{C}$ for 30 min. After completion of the reaction, monitored by TLC, the catalyst separated by centrifugation. Then the crude products were recrystallized from aqueous ethanol to get the final compounds $\left(A_{1}-A_{15}\right)($ Scheme 1$)$.

\section{Determination of anticonvulsant activity}

\section{Chemicals}

Pentylenetetrazole (PTZ) was purchased from Sigma (UK). It was dissolved in physiological saline solution and DMSO was used as co-solvent. Diazepam was purchased from Kimidaroo (Iran) and was disssolved in normal saline. In all experiments PTZ was administered intravenously (iv) and all other drugs were administered intraperitoneally (ip) 30 min before PTZ.

\section{Subjects}

Male NMRI mice (24-30 g, Shiraz University of Medical Sciences) were used throughout this study. The animals were housed in temperature controlled room $\left(24 \pm 1^{\circ} \mathrm{C}\right)$ on a $12 \mathrm{~h}$ light/dark cycle with free access to food and water. All the experiments were conducted between 9 and 15 o'clock each day. All procedures were carried out in accordance with institutional guidelines for animal care and use. Ethical guidelines for animal studies were confirmed by Vice chancellery for research affairs, Shiraz University of Medical Sciences. Each mouse was used only once and each treatment group consisted of six to eight animals.

Behavioral seizure evaluation

Page $11 / 19$ 
In intravenous PTZ experiments, animals received acute intraperitoneal injections of different doses of spirooxindole- $4 H$ pyran derivatives $(20,40,60$ or $80 \mathrm{mg} / \mathrm{kg}) 30 \mathrm{~min}$ before administration of PTZ. Diazepam was used as positive control. In this experimental model, a catheter with a $4.6 \mathrm{~mm}$ diameter was connected to the tail of animal through 30-gauge dental needle and a syringe pump (Harvard, USA) was used to deliver an exact amount of PTZ with the rate of $0.5 \mathrm{~cm}^{3} / \mathrm{min}$ through a catheter. The infusion was halted were forelimb clonus followed by full clonus of the body was observed. The minimal dose of PTZ (mg/kg of mice weight) needed to induce the clonic seizure, was considered as an index of seizure threshold.

\section{Statistical analysis}

Data are expressed as Mean \pm SEM. All the values were analyzed using the one-way analysis of variance (ANOVA) followed by Dunnett's test. Statistical software was SPSS (Ver18) and $p<0.05$ was considered statistically significant.

\section{Conclusions}

To conclude, simple methodology with high yields by organocatalyst (graphene oxide) based on 1-(2-aminoethyl) piperazine was applied for synthesis of spirooxindole-4H-pyran. The advantages of this method are described by efficient catalyst under ethanol as safe solvent and easy work-up. Also, fiftheen derivatives of spirooxindole-4H-pyran were selected to be screened for their anticonvulsant activity against subcutaneous PTZ induced seizures in mice. The results revealed that most of compounds (A2-A7) and $\left(A_{9}-A_{15}\right)$ significantly increased the seizure threshold. The results indicated that compounds $A_{3}$ and $A_{12}$ were choice to be the most active anticonvulsant agents in this study and also, exhibited reasonable physicochemical properties with good predicted drug-likeness values. The molecular docking simulation were done for all the synthesized compounds into the binding site of GABA-A target. There was desirable correlation between the results of docking studies and anticonvulsant activities. Therefore, we represented a new scaffold that could be further optimized for future development of more effective anticonvulsant agents.

\section{Declarations}

Acknowledgements

Financial assistance from the Shiraz University of Medical Sciences by way of grant number 95-01-36-13130 and 24020 are gratefully acknowledged.

\section{Conflicts of interest}

The authors declare no conflict of interest.

\section{Ethical approval}

All applicable international, national, and/or institutional guidelines for the care and use of animals were followed. All procedures performed in studies involving animals were in accordance with ethical standards of the institution or practice at which the studies were conducted.

\section{References}

1. Marzouk AA, Bass AK, Ahmed MS, Abdelhamid AA, Elshaier YA, Salman AM et al (2020) Design, synthesis and anticonvulsant activity of new imidazolidindione and imidazole derivatives. Bioorg Chem 101:104020

2. Kumar R, Singh T, Singh H, Jain S, Roy R (2014) Design, synthesis and anticonvulsant activity of some new 6, 8-halosubstituted-2h-[1, 2, 4] triazino [5, 6-b] indole-3 (5h)-one/-thione and 6, 8-halo-substituted 5-methyl-2h-[1, 2, 4] triazino [5, 6-b] indol-3 (5h)-one/-thione. EXCLI J 13:225

3. Tang DH, Malone DC, Warholak TL, Chong J, Armstrong EP, Slack MK et al (2015) Prevalence and incidence of epilepsy in an elderly and low-income population in the United States. Journal of clinical neurology 11(3):252-261

4. Banerjee B (2017) Recent developments on ultrasound-assisted one-pot multicomponent synthesis of biologically relevant heterocycles. Ultrason Sonochem 35:15-35

Page 12/19 
5. Cheng D, Ishihara Y, Tan B, Barbas CF III (2014) Organocatalytic asymmetric assembly reactions: synthesis of spirooxindoles via organocascade strategies. Acs Catalysis 4(3):743-762

6. Pavlovska TL, Redkin RG, Lipson VV, Atamanuk DV (2016) Molecular diversity of spirooxindoles. Synthesis and biological activity. Mol Diversity 20(1):299-344

7. Akbari A, Azami-Sardooei Z, Hosseini-Nia A (2013) Synthesis and biological evaluation of 2-Amino-4H-pyran-3, 4, 5tricarboxylate salt derivatives. J Korean Chem Soc 57(4):455-460

8. Laskar S, Brahmachari G (2014) Access to biologically relevant diverse chromene heterocycles via multicomponent reactions (MCRs): Recent advances. Organic Biomolecular Chemistry 2:1-50

9. Mahdavi S, Habibi A, Dolati H, Habibi M. Synthesis of 2-amino-4H-pyran derivatives via three component reaction in the presence of 4-Dimethylaminopyridine (DMAP) as catalyst. 2014

10. Brahmachari G (2021) Green synthetic approaches for biologically relevant 2-amino-4H-pyrans and 2-amino-4H-pyranannulated heterocycles in aqueous media. Green Synthetic Approaches for Biologically Relevant Heterocycles. Elsevier, pp 471-504

11. Martínez-Grau A, Marco J (1997) Friedländer reaction on 2-amino-3-cyano-4H-pyrans: synthesis of derivatives of 4H-pyran [2, 3b] quinoline, new tacrine analogues. Bioorg Med Chem Lett 7(24):3165-3170

12. Vine KL, Locke JM, Ranson M, Pyne SG, Bremner JB (2007) An investigation into the cytotoxicity and mode of action of some novel N-alkyl-substituted isatins. Journal of medicinal chemistry 50(21):5109-5117

13. Logan J, Fox M, Morgan J, Makohon A, Pfau C (1975) Arenavirus inactivation on contact with N-substituted isatin betathiosemicarbazones and certain cations. J Gen Virol 28(3):271-283

14. Pandeya SN, Raja AS, Stables JP (2002) Synthesis of isatin semicarbazones as novel anticonvulsants-role of hydrogen bonding. J Pharm Pharm Sci 5(3):266-271

15. Pandeya SN, Smitha S, Jyoti M, Sridhar SK (2005) Biological activities of isatin and its derivatives. Acta Pharm 55(1):27-46

16. Divar M, Zomorodian K, Sabet R, Moeini M, Khabnadideh S. An Efficient Method for Synthesis of Some Novel Spirooxindole4H-Pyran Derivatives. Polycycl Aromat Compd. 2019:1-14

17. Mohammadi Ziarani G, Hosseini Mohtasham N, Lashgari N, Badiei A, Amanlou M, Bazl R (2012) Convenient one-pot synthesis of spirooxindole-4H-pyrans in the presence of SBA-Pr-NH2 and evaluation of their urease inhibitory activities. J Nanostruct 2(4):489-500

18. Wang L-M, Jiao N, Qiu J, Yu J-J, Liu J-Q, Guo F-L et al (2010) Sodium stearate-catalyzed multicomponent reactions for efficient synthesis of spirooxindoles in aqueous micellar media. Tetrahedron 66(1):339-343

19. Li Y, Chen H, Shi C, Shi D, Ji S (2010) Efficient one-pot synthesis of spirooxindole derivatives catalyzed by I-proline in aqueous medium. J Comb Chem 12(2):231-237

20. Zhu S-L, Ji S-J, Zhang Y (2007) A simple and clean procedure for three-component synthesis of spirooxindoles in aqueous medium. Tetrahedron 63(38):9365-9372

21. Shanthi G, Subbulakshmi G, Perumal PT (2007) A new InCl3-catalyzed, facile and efficient method for the synthesis of spirooxindoles under conventional and solvent-free microwave conditions. Tetrahedron 63(9):2057-2063

22. Bahrami K, Khodaei MM, Fattahpour P (2011) SBA-15-Pr-SO $3 \mathrm{H}$ as nanoreactor catalyzed oxidation of sulfides into sulfoxides. Catal Sci Technol 1(3):389-393

23. Didaskalou C, Kupai J, Cseri L, Barabas J, Vass E, Holtzl T et al (2018) Membrane-grafted asymmetric organocatalyst for an integrated synthesis-separation platform. ACS Catalysis 8(8):7430-7438

24. Zhu Y, Murali S, Cai W, Li X, Suk JW, Potts JR et al (2010) Graphene and graphene oxide: synthesis, properties, and applications. Advanced materials 22(35):3906-3924

25. Singh RK, Kumar R, Singh DP (2016) Graphene oxide: strategies for synthesis, reduction and frontier applications. Rsc Advances 6(69):64993-65011

26. Kwan YCG, Ng GM, Huan CHA (2015) Identification of functional groups and determination of carboxyl formation temperature in graphene oxide using the XPS 0 1s spectrum. Thin Solid Films 590:40-48 
27. Wang S, Li X, Liu Y, Zhang C, Tan X, Zeng G et al (2018) Nitrogen-containing amino compounds functionalized graphene oxide: synthesis, characterization and application for the removal of pollutants from wastewater: a review. J Hazard Mater 342:177191

28. Mohammadi-Khanaposhtani M, Shabani M, Faizi M, Aghaei I, Jahani R, Sharafi Z et al (2016) Design, synthesis, pharmacological evaluation, and docking study of new acridone-based 1, 2, 4-oxadiazoles as potential anticonvulsant agents. Eur J Med Chem 112:91-98

29. Mohammadi-Khanaposhtani M, Shabani M, Faizi M, Aghaei I, Jahani R, Sharafi Z et al (2016) Design, synthesis, pharmacological evaluation, and docking study of new acridone-based 1, 2, 4-oxadiazoles as potential anticonvulsant agents. Eur J Med Chem 112:91-98

30. Asadollahi A, Asadi M, Hosseini FS, Ekhtiari Z, Biglar M, Amanlou M (2019) Synthesis, molecular docking, and antiepileptic activity of novel phthalimide derivatives bearing amino acid conjugated anilines. Res Pharm Sci 14(6):534

31. Enna SJ (2007) The GABA receptors. The GABA receptors. Springer, pp 1-21

32. Watanabe M, Maemura K, Kanbara K, Tamayama T, Hayasaki H (2002) GABA and GABA Receptors in the Central Nervous System and Other Organs. In: Jeon KW (ed) International Review of Cytology, 213. Academic Press, pp 1-47

\section{Figures}

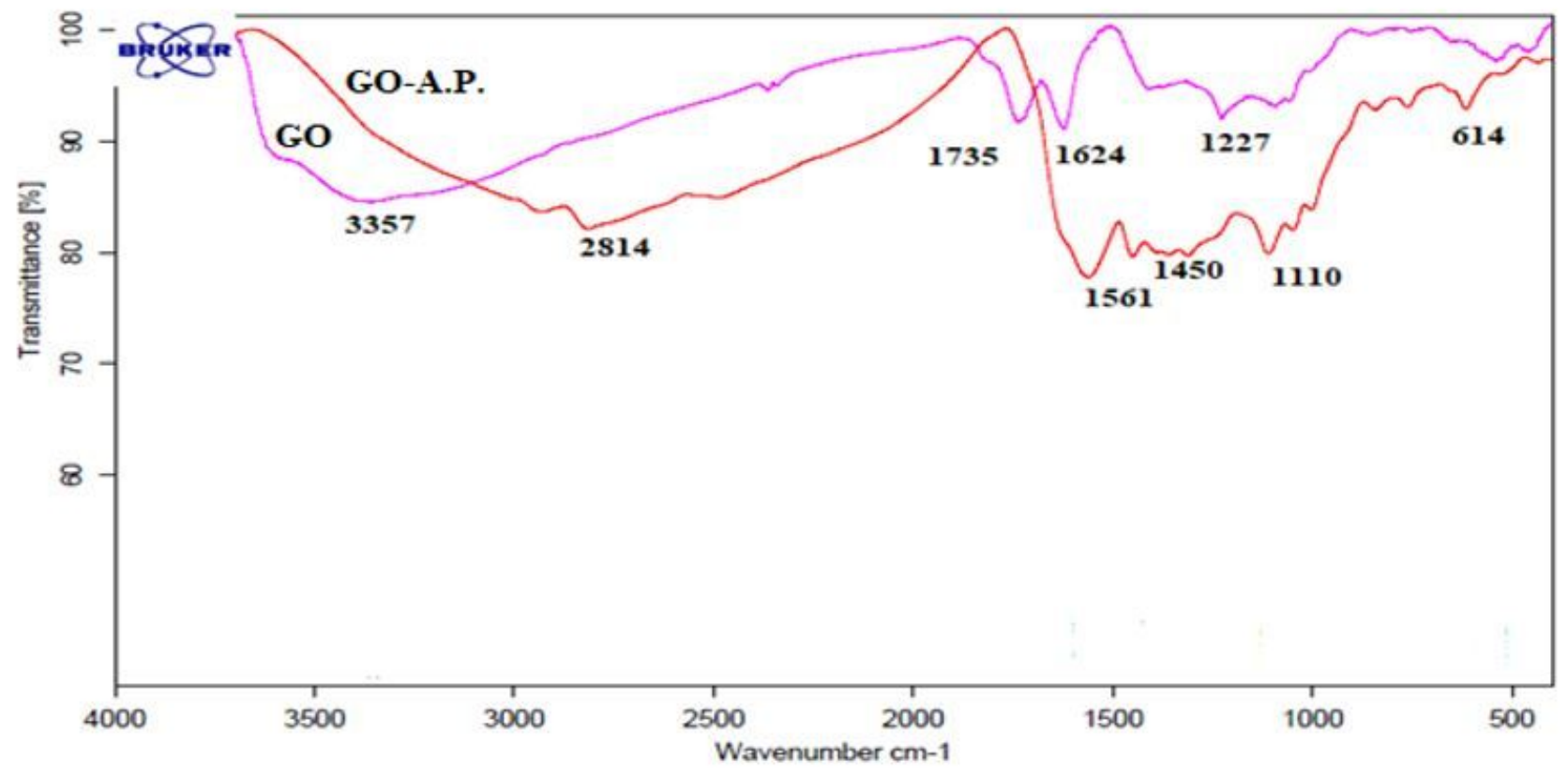

\section{Figure 1}

FT-IR spectrum of GO and GO-A.P. 


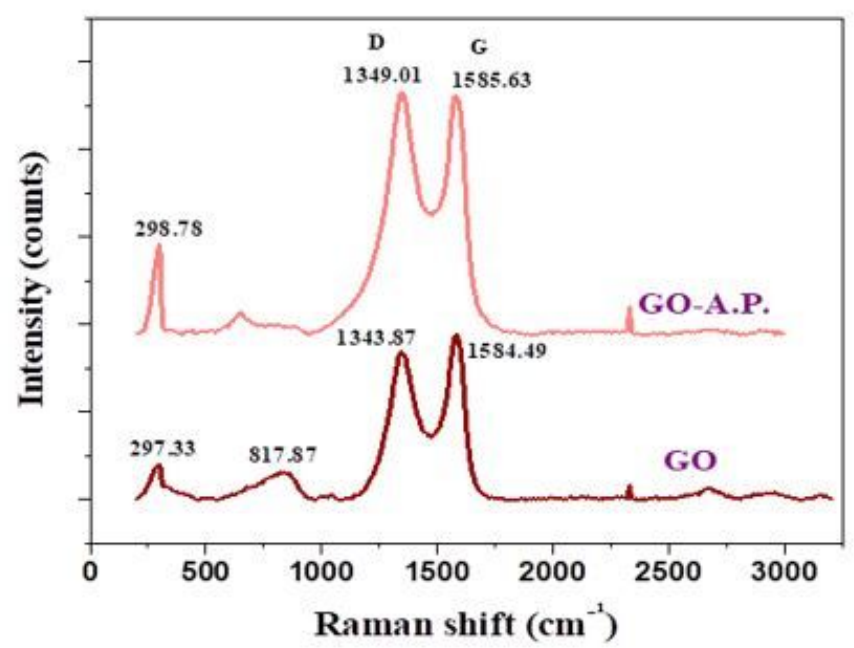

Figure 2

Raman spectra of GO and GO-A.P.

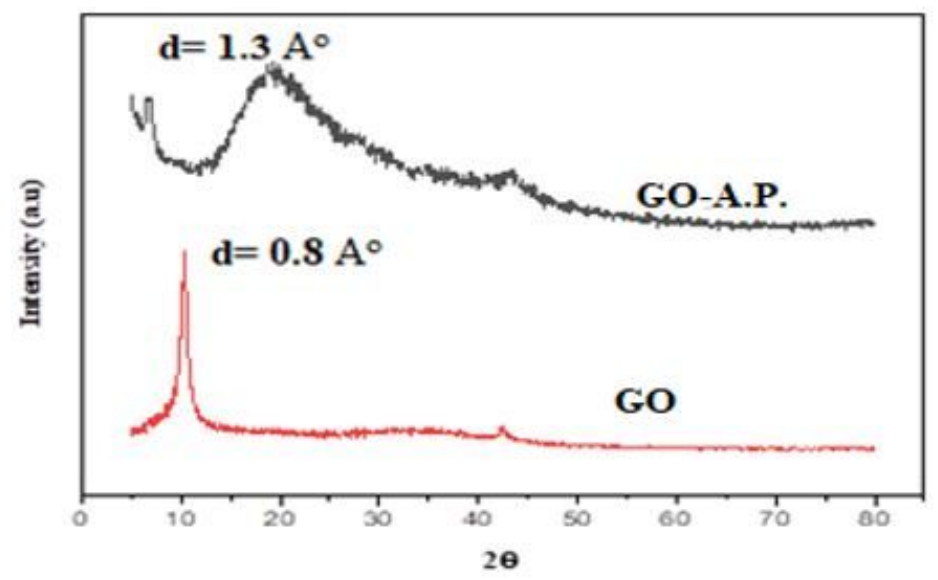

Figure 3

XRD patterns of $\mathrm{GO}$ and GO-A.P. 


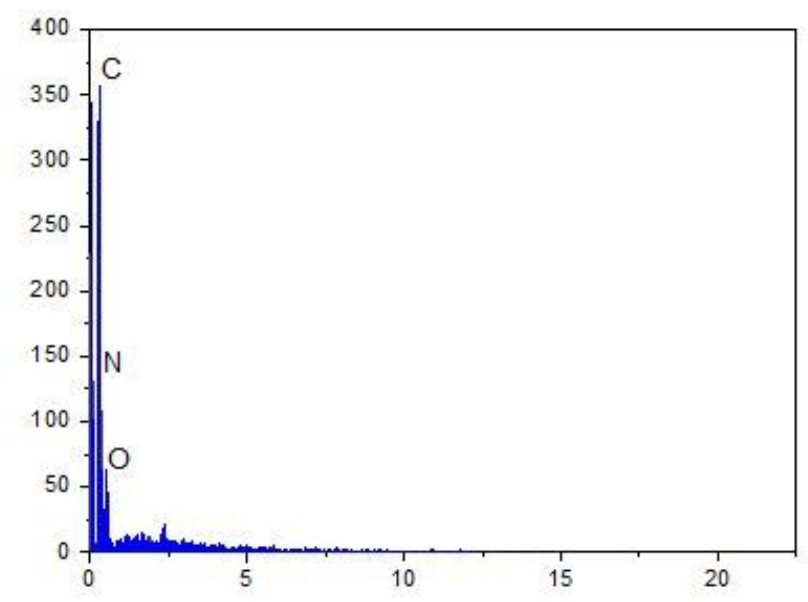

\section{Figure 4}

EDS analysis of GO-A.P. C= $50.41 \%, \mathrm{~N}=30.27 \%, \mathrm{O}=19.31 \%$
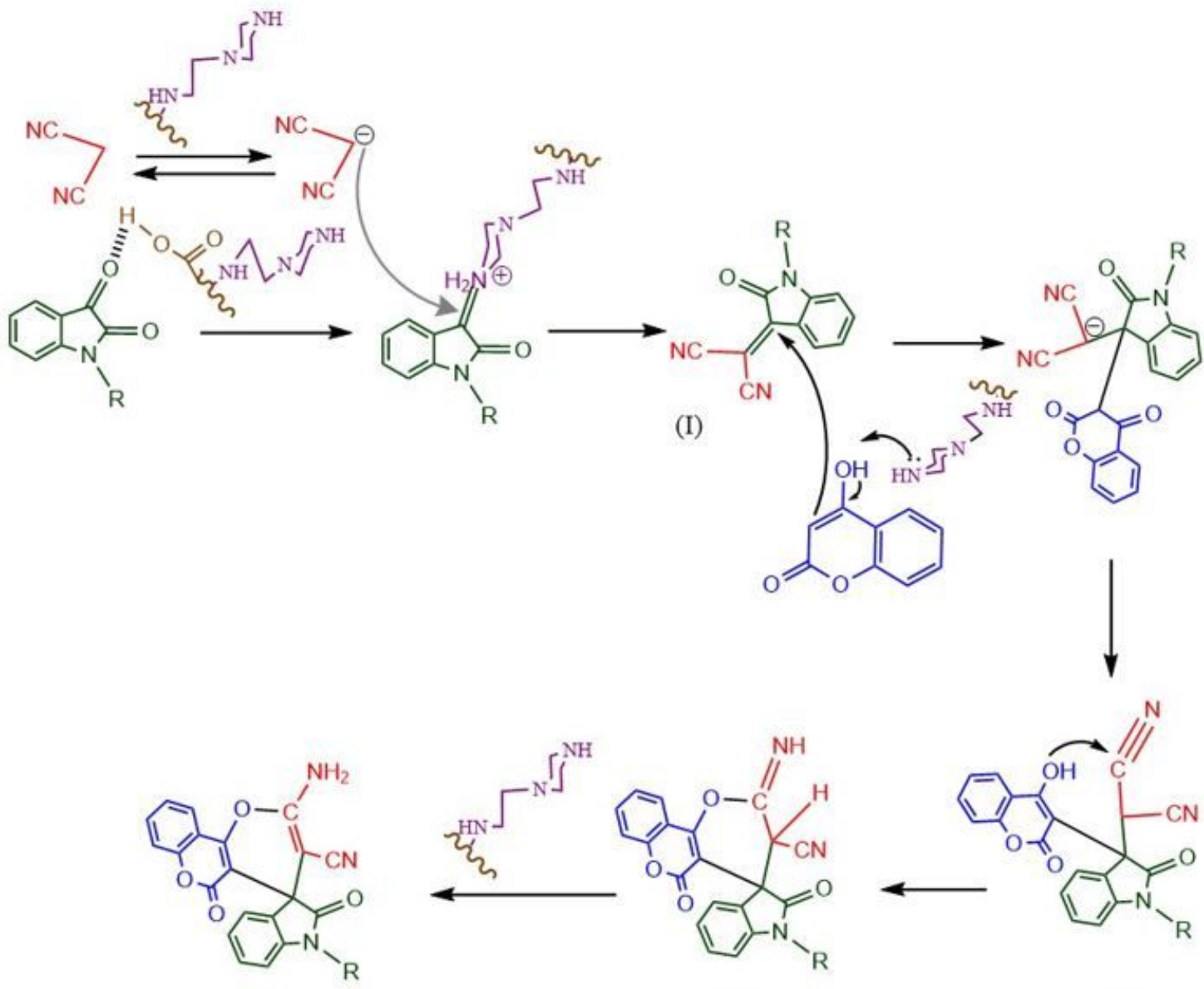

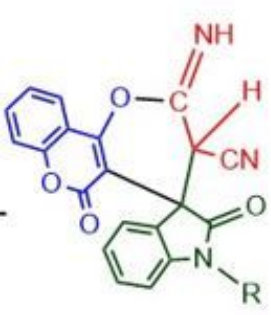

(III)

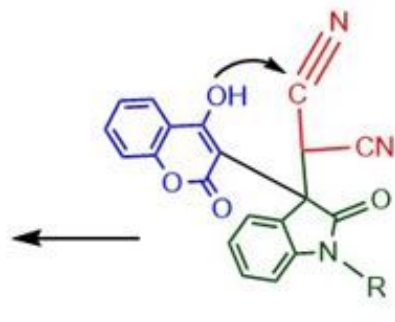

(II)

\section{Figure 5}

Proposed mechanism for synthesis of spirooxindole-4H-pyran derivatives 


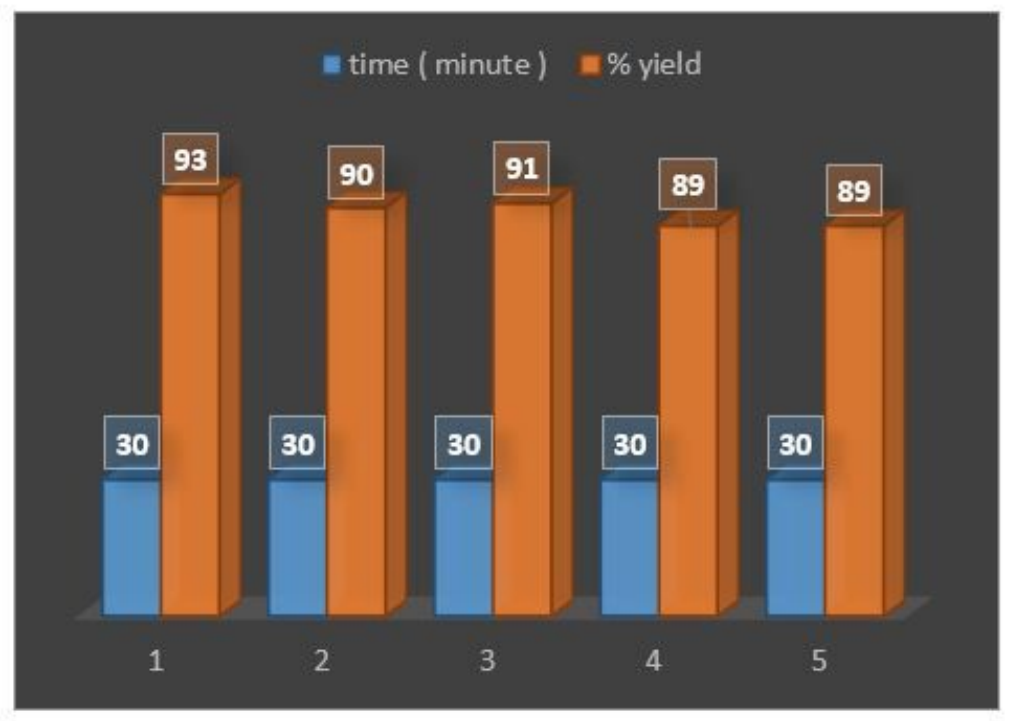

Figure 6

Recyclability of the catalyst (GO-A.P.) in the synthesis of A2
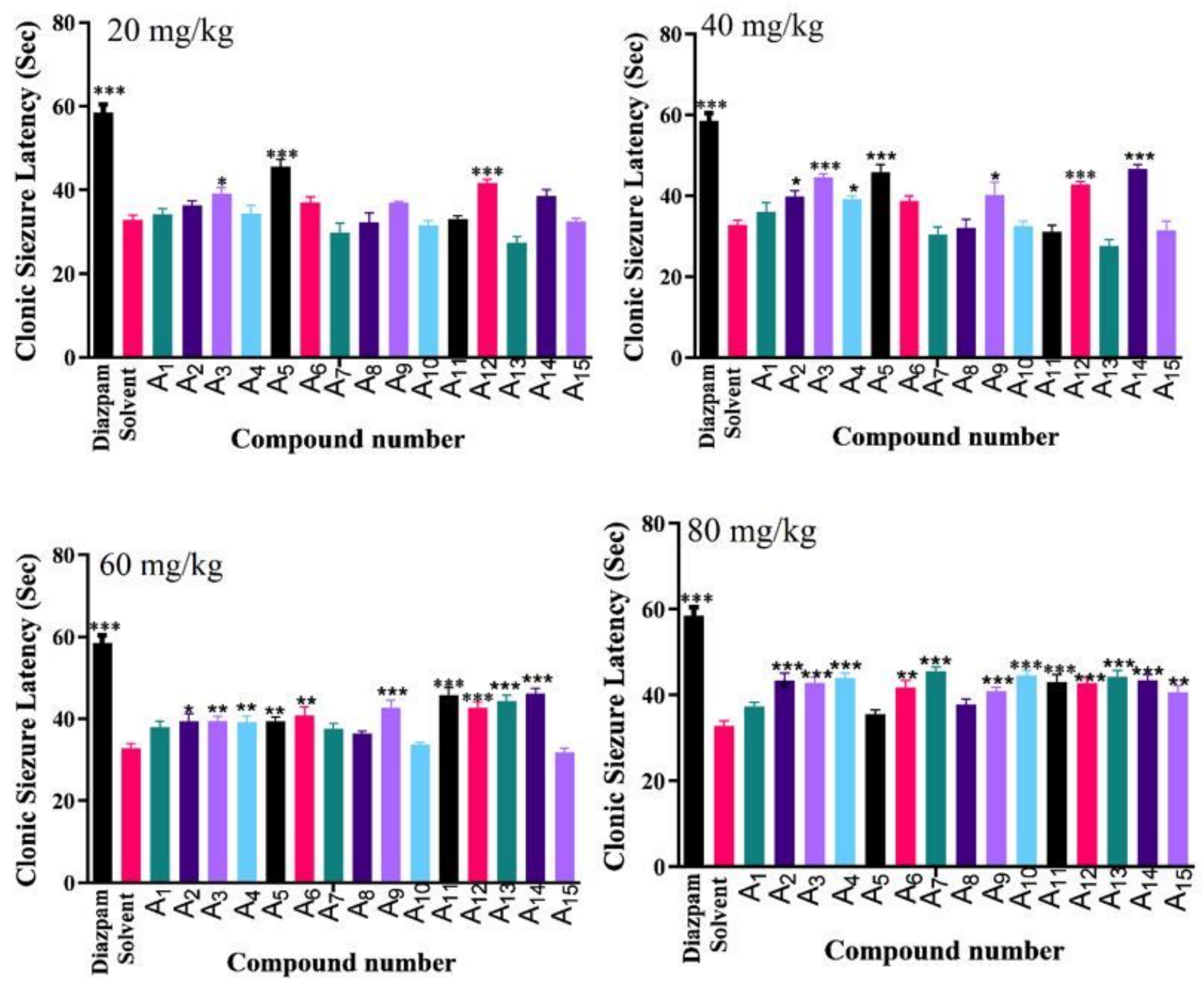
Figure 7

Effect of spirooxindole-4H-pyran derivatives on clonic seizure latency. Data are mean \pm standard error of the mean of the latency time, $(n=6-8) .{ }^{*} P<0.05,{ }^{*} P<0.01$ and $* * * P<0.001$
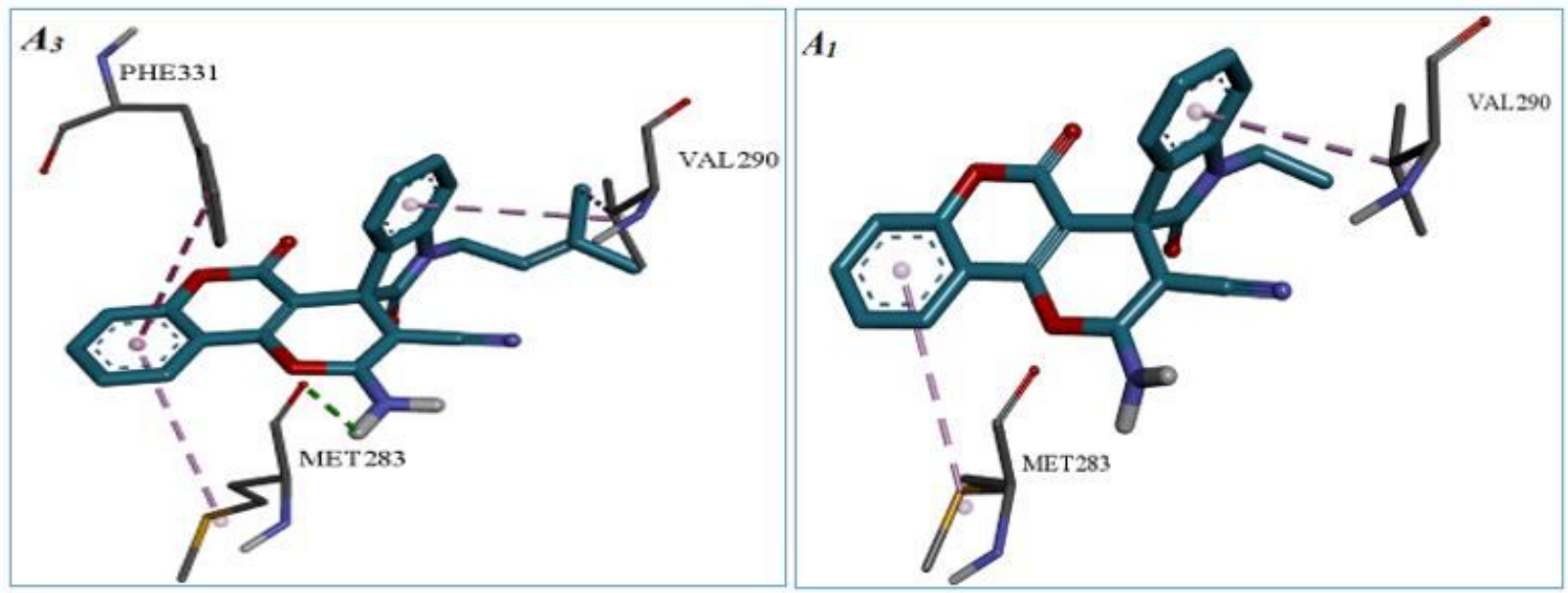

Figure 8

Interactions of $\mathrm{A} 3$ and $\mathrm{A} 1$ with the residues in the binding site of $6 \mathrm{X} 3 \mathrm{X}$ receptor

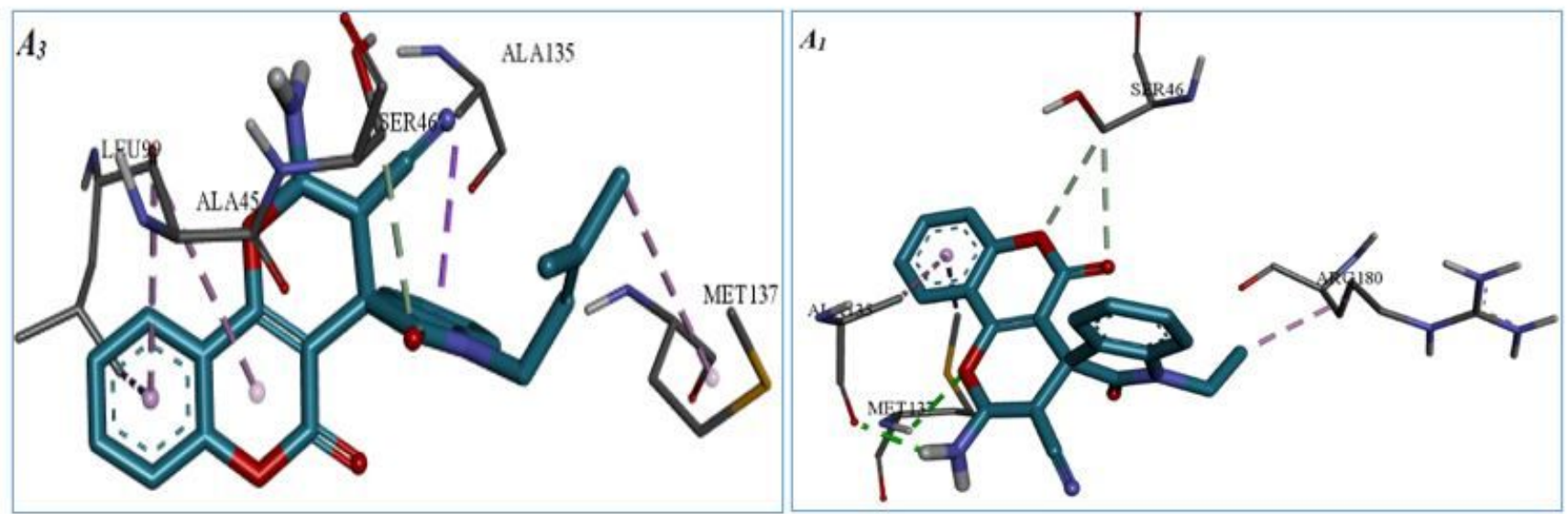

Figure 9

Interactions of $\mathrm{A} 3$ and $\mathrm{A} 1$ with the residues in the binding site of $4 \mathrm{COF}$ receptor 


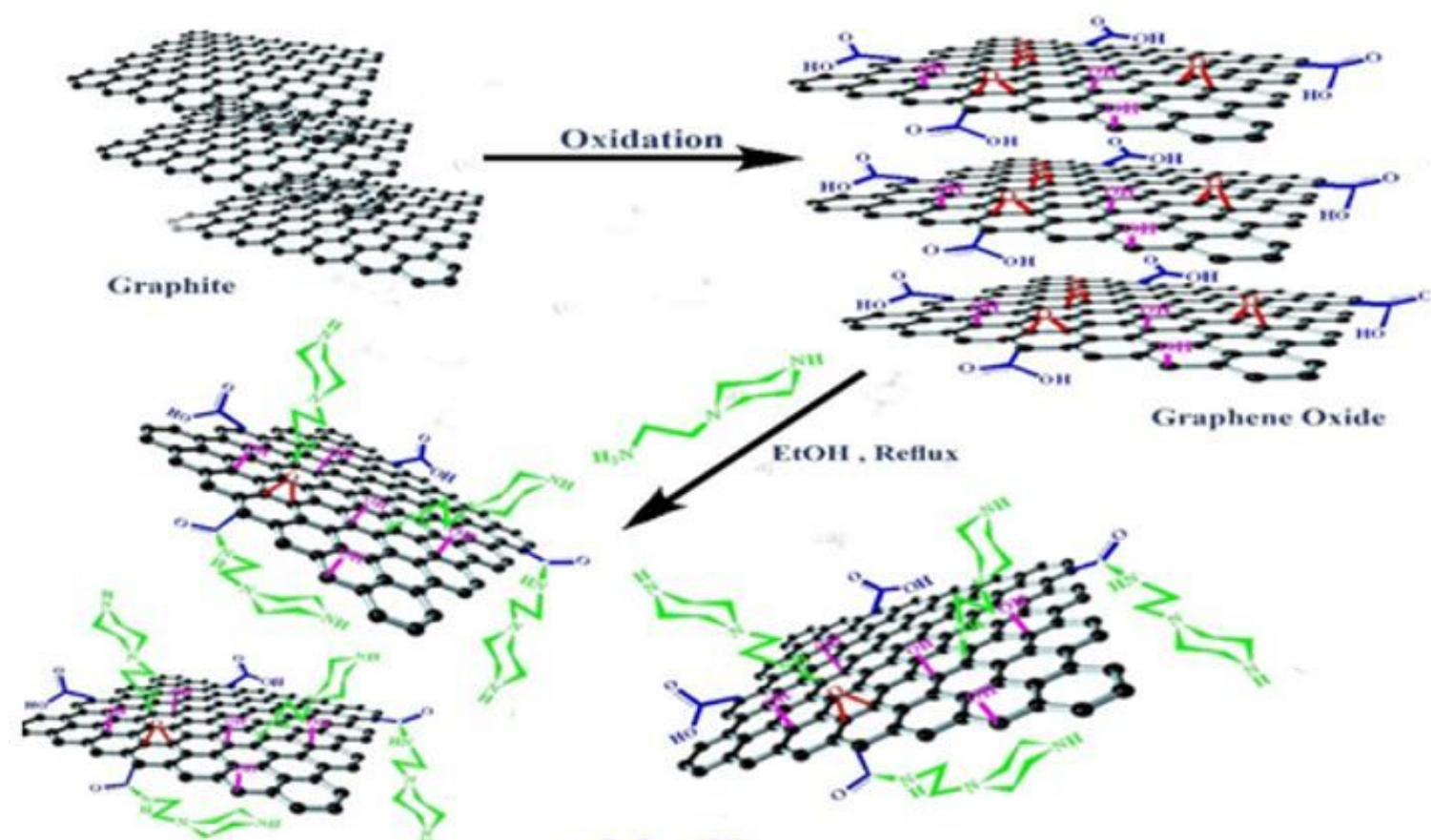

GO-A.P.

Figure 10

Synthesis and functionalization of GO with 1(2-aminoethyl) piperazine

\section{Supplementary Files}

This is a list of supplementary files associated with this preprint. Click to download.

- supplementary.docx 